\title{
STABILIZATION OF A RIGID BODY MOVING IN A COMPRESSIBLE VISCOUS FLUID
}

\author{
ARNAB ROY AND TAKÉO TAKAHASHI \\ Université de Lorraine, CNRS, Inria, IECL, F-54000 Nancy, France
}

\begin{abstract}
We consider the stabilizability of a fluid-structure interaction system where the fluid is viscous and compressible and the structure is a rigid ball. The feedback control of the system acts on the ball and corresponds to a force that would be produced by a spring and a damper connecting the center of the ball to a fixed point $h_{1}$. We prove the global-in-time existence of strong solutions for the corresponding system under a smallness condition on the initial velocities and on the distance between the initial position of the center of the ball and $h_{1}$. Then, we show with our feedback law, that the fluid and the structure velocities go to 0 and that the center of the ball goes to $h_{1}$ as $t \rightarrow \infty$.
\end{abstract}

Keywords. Fluid-structure interaction, compressible Navier-Stokes system, global solutions, stabilization

AMS subject classifications. 35Q35, 35D30, 35D35, 35R37, 76N10, 93D15, 93D20.

\section{Contents}

1. Introduction and main result

Notation

2. Local in time existence of solutions

2.1. Lagrangian change of variables

2.2. Analysis of a linear problem

2.3. Estimates of the nonlinear terms

2.4. Proof of Theorem 2.1 14

3. Global in time existence of solutions 15

3.1. A priori estimates $\quad 15$

3.2. Proof of Theorem $1.1 \quad 22$

4. Proof of Theorem $1.2 \quad 24$

$\begin{array}{ll}\text { References } & 27\end{array}$

\section{INTRODUCTION AND MAIN RESULt}

Let $\Omega \subset \mathbb{R}^{3}$ be a bounded domain with $C^{4}$ boundary occupied by a fluid and a rigid body. We denote by $\mathcal{B}(t) \subset \Omega$, the domain of the rigid body and we assume it is an open ball of radius 1 and of center $h(t)$, where $t \in \mathbb{R}_{+}$is the time variable. We suppose that the fluid domain $\mathcal{F}(t)=\Omega \backslash \overline{\mathcal{B}(t)}$ is connected.

Date: October 17, 2019. 
The fluid is modeled by the compressible Navier-Stokes system whereas the motion of the rigid body is governed by the balance equations for linear and angular momentum. We also assume the no-slip boundary conditions. The equations of motion of fluid-structure are:

$$
\begin{gathered}
\frac{\partial \rho}{\partial t}+\operatorname{div}(\rho u)=0 \quad t>0, x \in \mathcal{F}(t), \\
\rho\left(\frac{\partial u}{\partial t}+(u \cdot \nabla) u\right)-\operatorname{div} \sigma(u, p)=0 \quad t>0, x \in \mathcal{F}(t), \\
m \ell^{\prime}=-\int_{\partial \mathcal{B}(t)} \sigma(u, p) N d \Gamma+w \quad t \geqslant 0, \\
J \omega^{\prime}=-\int_{\partial \mathcal{B}(t)}(x-h(t)) \times \sigma(u, p) N d \Gamma \quad t \geqslant 0, \\
h^{\prime}=\ell \quad t \geqslant 0, \\
u(t, x)=0 \quad t>0, x \in \partial \Omega, \\
u(t, x)=\ell(t)+\omega(t) \times(x-h(t)) \quad t>0, x \in \partial \mathcal{B}(t), \\
\rho(0, \cdot)=\rho_{0}, \quad u(0, \cdot)=u_{0} \quad \text { in } \mathcal{F}(0), \\
h(0)=h_{0}, \quad \ell(0)=\ell_{0}, \quad \omega(0)=\omega_{0} .
\end{gathered}
$$

In the above equations, $\rho=\rho(t, x)$ and $u=u(t, x)$ represent respectively the density and the velocity of the fluid and the pressure of the fluid is denoted by $p$. We assume that the flow is in the barotropic regime and we focus on the isentropic case where the relation between $p$ and $\rho$ is given by the constitutive law:

$$
p=a \rho^{\gamma},
$$

with $a>0$ and the adiabatic constant $\gamma>\frac{3}{2}$. The Cauchy stress tensor is defined as:

$$
\sigma(u, p)=2 \mu \mathbb{D}(u)+\lambda \operatorname{div} u \mathbb{I}_{3}-p \mathbb{I}_{3},
$$

where $\mathbb{D}(u)=\frac{1}{2}\left(\nabla u+\nabla u^{\top}\right)$ denotes the symmetric part of the velocity gradient $\left(\nabla u^{\top}\right.$ is the transpose of the matrix $\nabla u$ ) and $\lambda, \mu$ are the viscosity coefficients satisfying

$$
\mu>0, \quad \lambda+\mu \geqslant 0
$$

Here $\ell$ and $\omega$ are the linear and angular velocities of the rigid body, $N(t, x)$ is the unit normal to $\partial \mathcal{B}(t)$ at the point $x \in \partial \mathcal{B}(t)$, directed to the interior of the ball and $m, J$ are the mass and the moment of inertia of the rigid ball respectively. The formulae for $m$ and $J$ are

$$
m=\frac{4}{3} \pi \rho_{\mathcal{B}}, \quad J=\frac{2 m}{5} \mathbb{I}_{3},
$$

where $\rho_{\mathcal{B}}>0$ is the constant density of the rigid ball.

Finally, $w$ (in (1.3)) is our control that we take as a feedback control:

$$
w(t)=k_{p}(t)\left(h_{1}-h(t)\right)-k_{d} \ell(t),
$$

where $k_{d} \geqslant 0$ and $k_{p}(t) \geqslant 0$ are well-chosen so that

$$
\lim _{t \rightarrow \infty} h(t)=h_{1}
$$


whereas the velocities of the fluid and of the rigid ball go to 0:

$$
\lim _{t \rightarrow \infty} u(t)=0, \quad \lim _{t \rightarrow \infty} \ell(t)=0, \quad \lim _{t \rightarrow \infty} \omega(t)=0 .
$$

In literature, this type of control is known as Proportional-Derivative (PD) controller generated by a spring and a damper. The spring-damper is connected from the center of the ball to the fixed anchor point $h_{1}$ and it is attracting the ball towards the point $h_{1}$.

In order to give the precise statement of stabilization (Theorem 1.2), we first need a global in time existence result for (1.1)-(1.10) with (1.10). Such a result in the case without control is given in [1] by adapting a method introduced in [13].

Here we will prove again this existence result, with the same approach but with a special attention to the estimates on $h(t)$ and with some modifications in the proof of [1] due to the feedback law (1.10).

In order to state our result we introduce $\bar{\rho}$ the mean-value of $\rho_{0}$ :

$$
\bar{\rho}=\frac{1}{|\mathcal{F}(0)|} \int_{\mathcal{F}(0)} \rho_{0}(x) d x .
$$

Note that, from equation (1.1) and Reynold's Transport Theorem, we obtain

$$
\int_{\mathcal{F}(0)} \rho_{0}(x) d x=\int_{\mathcal{F}(t)} \rho(t, x) d x .
$$

For $0 \leqslant T_{1}<T_{2} \leqslant \infty$, we introduce the following space:

$$
\begin{gathered}
\widehat{\mathcal{S}}_{T_{1}, T_{2}}=\left\{(\rho, u, \ell, \omega) \mid \rho \in L^{2}\left(T_{1}, T_{2} ; H^{3}(\mathcal{F}(t))\right) \cap B C^{0}\left(\left[T_{1}, T_{2}\right] ; H^{3}(\mathcal{F}(t))\right) \cap H^{1}\left(T_{1}, T_{2} ; H^{2}(\mathcal{F}(t))\right.\right. \\
\cap B C^{1}\left(\left[T_{1}, T_{2}\right] ; H^{2}(\mathcal{F}(t))\right) \cap H^{2}\left(T_{1}, T_{2} ; L^{2}(\mathcal{F}(t))\right), \\
u \in L^{2}\left(T_{1}, T_{2} ; H^{4}(\mathcal{F}(t))\right) \cap B C^{0}\left(\left[T_{1}, T_{2}\right] ; H^{3}(\mathcal{F}(t))\right) \cap H^{1}\left(T_{1}, T_{2} ; H^{2}(\mathcal{F}(t))\right. \\
\cap B C^{1}\left(\left[T_{1}, T_{2}\right] ; H^{1}(\mathcal{F}(t))\right) \cap H^{2}\left(T_{1}, T_{2} ; L^{2}(\mathcal{F}(t))\right), \\
\left.\ell \in H^{2}\left(T_{1}, T_{2}\right), \quad \omega \in H^{2}\left(T_{1}, T_{2}\right)\right\} .
\end{gathered}
$$

Here $B C^{k}$ are the functions of class $C^{k}$ bounded with bounded derivatives. We set

$$
\begin{gathered}
\|(\rho, u, \ell, \omega)\|_{\widehat{\mathcal{S}}_{T_{1}, T_{2}}}=\|\rho-\bar{\rho}\|_{L^{\infty}\left(T_{1}, T_{2} ; H^{3}(\mathcal{F}(t))\right)}+\|\rho-\bar{\rho}\|_{H^{1}\left(T_{1}, T_{2} ; H^{2}(\mathcal{F}(t))\right)}+\|\rho-\bar{\rho}\|_{W^{1, \infty}\left(T_{1}, T_{2} ; H^{2}(\mathcal{F}(t))\right)} \\
+\|\rho-\bar{\rho}\|_{H^{2}\left(T_{1}, T_{2} ; L^{2}(\mathcal{F}(t))\right)}+\|u\|_{L^{2}\left(T_{1}, T_{2} ; H^{4}(\mathcal{F}(t))\right)}+\|u\|_{L^{\infty}\left(T_{1}, T_{2} ; H^{3}(\mathcal{F}(t))\right)}+\|u\|_{H^{1}\left(T_{1}, T_{2} ; H^{2}(\mathcal{F}(t))\right)} \\
+\|u\|_{W^{1, \infty}\left(T_{1}, T_{2} ; H^{1}(\mathcal{F}(t))\right)}+\|u\|_{H^{2}\left(T_{1}, T_{2} ; L^{2}(\mathcal{F}(t))\right)}+\|\ell\|_{H^{2}\left(T_{1}, T_{2}\right)}+\|\ell\|_{W^{1, \infty}\left(T_{1}, T_{2}\right)} \\
+\|\omega\|_{H^{2}\left(T_{1}, T_{2}\right)}+\|\omega\|_{W^{1, \infty}\left(T_{1}, T_{2}\right)},
\end{gathered}
$$

and for $T>0$

$$
\left\|\left(\rho_{0}, u_{0}, \ell_{0}, \omega_{0}\right)\right\|_{\widehat{\mathcal{S}}_{T, T}}=\left\|\rho_{0}-\bar{\rho}\right\|_{H^{3}(\mathcal{F}(T))}+\left\|u_{0}\right\|_{H^{3}(\mathcal{F}(T))}+\left|\ell_{0}\right|+\left|\omega_{0}\right| .
$$

Since we are working with regular solutions of (1.1)-(1.10), we need to introduce the following compatibility conditions at initial time:

$$
\begin{gathered}
u_{0}(y)=\ell_{0}+\omega_{0} \times\left(y-h_{0}\right) \text { for } y \in \partial \mathcal{B}(0), \quad u_{0}=0 \text { on } \partial \Omega, \\
-\frac{1}{\rho_{0}} \operatorname{div} \sigma\left(u_{0}, p_{0}\right)=0 \text { on } \partial \Omega
\end{gathered}
$$




$$
\begin{aligned}
&-\left(\omega_{0}\right.\left.\times\left(\omega_{0} \times\left(y-h_{0}\right)\right)\right)-\frac{1}{\rho_{0}} \operatorname{div} \sigma\left(u_{0}, p_{0}\right)(y) \\
&=\frac{1}{m}\left[\int_{\partial \mathcal{B}(0)} \sigma\left(u_{0}, p_{0}\right) n d \Gamma-k_{d} \ell_{0}\right]+\left[J^{-1} \int_{\partial \mathcal{B}(0)}\left(x-h_{0}\right) \times \sigma\left(u_{0}, p_{0}\right) n d \Gamma_{x}\right] \times\left(y-h_{0}\right) \\
& \text { for } y \in \partial \mathcal{B}(0),
\end{aligned}
$$

where

Finally, we introduce the following notation

$$
p_{0}=a \rho_{0}^{\gamma}
$$

$$
\Omega^{0}:=\{x \in \Omega ; \operatorname{dist}(x, \partial \Omega)>1\} .
$$

Our hypotheses on $k_{p}$ and $k_{d}$ are the following ones:

$$
k_{p} \in C^{1}\left(\mathbb{R}_{+},[0,1]\right), k_{p}(0)=0, k_{p}>0 \text { in }(0, \infty), k_{p} \equiv 1 \text { in }\left[T_{I}, \infty\right), 0 \leqslant k_{p}^{\prime}<\frac{k_{d}}{2 T_{I}^{2}}
$$

for some $T_{I}>0$.

Theorem 1.1. Assume that $\Omega^{0}$ is non empty and connected. Let $h_{1} \in \Omega^{0}$ and $\bar{\rho}>0$. Assume $w$ is given by the feedback law (1.10) with $\left(k_{p}, k_{d}\right)$ satisfying (1.17). There exists $\delta>0$ such that for any

$$
h_{0} \in \Omega^{0}, \rho_{0} \in H^{3}(\mathcal{F}(0)), \rho_{0}>0, u_{0} \in H^{3}(\mathcal{F}(0)), \ell_{0}, \omega_{0} \in \mathbb{R}^{3},
$$

satisfying the compatibility conditions (1.14)-(1.16) with

$$
\left\|\left(\rho_{0}, u_{0}, \ell_{0}, \omega_{0}\right)\right\|_{\widehat{\mathcal{S}}_{0,0}}+\left|h_{1}-h_{0}\right| \leqslant \delta
$$

the system (1.1)-(1.10) admits a unique strong solution $(\rho, u, \ell, \omega) \in \widehat{\mathcal{S}}_{0, \infty}, h \in L^{\infty}(0, \infty)$. Moreover, there exist $C, \eta>0$ such that

$$
\begin{gathered}
\|(\rho, u, \ell, \omega)\|_{\widehat{\mathcal{S}}_{0, \infty}}+\left\|\sqrt{k_{p}}\left(h_{1}-h\right)\right\|_{L^{\infty}(0, \infty)} \leqslant C\left(\left\|\left(\rho_{0}, u_{0}, \ell_{0}, \omega_{0}\right)\right\|_{\widehat{\mathcal{S}}_{0,0}}+\left|h_{1}-h_{0}\right|\right), \\
\operatorname{dist}(h(t), \partial \Omega)>1+\eta \quad(t \geqslant 0) .
\end{gathered}
$$

We are now in a position to state our stabilization result.

Theorem 1.2. With the notations and assumptions of Theorem 1.1, the solution $(\rho, u, h, \ell, \omega)$ of (1.1)-(1.10) satisfies

$$
\begin{array}{r}
\lim _{t \rightarrow \infty}\|\rho(t, \cdot)-\bar{\rho}\|_{H^{2}(\mathcal{F}(t))}=0, \quad \lim _{t \rightarrow \infty}\|u(t, \cdot)\|_{H^{2}(\mathcal{F}(t))}=0, \\
\lim _{t \rightarrow \infty} h(t)=h_{1}, \quad \lim _{t \rightarrow \infty} \ell(t)=0, \quad \lim _{t \rightarrow \infty} \omega(t)=0 .
\end{array}
$$

During the last two decades, there has been a considerable interest in fluid-structure interaction problems involving moving interfaces. Broadly speaking, these types of models can be classified into two types: either the structure is moving inside the fluid or the structure is located at the boundary of the fluid domain. Since in this article we are interested in studying the motion of body inside the compressible fluid domain, below we mention related works from the literature concerning this case only.

The global-in-time existence (up to contact) of weak solutions for compressible viscous flow (for $\gamma \geqslant 2$ ) in a bounded domain of $\mathbb{R}^{3}$ interacting with a finite number of rigid bodies has been studied by Desjardins and Esteban [6]. In [9], Feireisl established the global existence result (for $\gamma>3 / 2$ ) regardless of possible collisions of several rigid bodies or a contact of the rigid bodies with the exterior 
boundary. Regarding strong solutions, the existence and uniqueness of global solutions for small initial data have been achieved in [1] in the Hilbert space framework by Boulakia and Guerrero as long as no collisions occur. Their work is based on a method proposed in [13] for a viscous compressible fluid (without structure). In a $L^{p}$ - $L^{q}$ setting, the authors in [12] proved the existence and uniqueness of local-in-time strong solutions for the system composed by rigid bodies immersed into a viscous compressible fluid and in [11], the authors establish the global in time existence up to contact.

Let us mention some works related to the large time behavior of fluid-structure interaction system. In [17], the authors analyze the fluid-structure model in one space dimension where the fluid is governed by the viscous Burgers equation and the solid mass is moving by the difference of pressure at both sides of it. They obtain that the asymptotic profile of the fluid is a self-similar solution of the Burgers equation and the point mass enjoys the parabolic trajectory as $t \rightarrow \infty$. An extension of this work in several space dimensions is obtained in [14] for the heat equations in interaction with a rigid body. Their result is that as $t \rightarrow \infty$, the fluid solution behaves as the fundamental solution of the heat equation and the ball goes to infinity in bidimensional case whereas the ball remains in a bounded domain in three dimension. Regarding the long-time behavior of a moving particle inside a NavierStokes fluid, the authors in [10] consider in particular the case of a ball falling over an horizontal plane and show that the velocity of the fluid goes to zero and the particle reaches the bottom of the container asymptotically in time. In [7], the authors analyze the case of a rigid disk immersed into a two-dimensional Navier-Stokes equations filling the exterior of the structure domain. They restrict to the case of a solid and a fluid with the same density and for the linear case.

Finally, let us mention two works using a control supported on the rigid body: [5] in the $1 \mathrm{~d}$ case for a Burgers-particle system and [16] in the $3 \mathrm{~d}$ case for a rigid ball moving into a viscous incompressible fluid. The main difference between this study and the two previous references come from the fact that in our case we need to deal with stronger solutions than in the incompressible case. In particular, to avoid compatibility conditions at $t=0$ that involve the feedback control $w$, we take here $k_{p}$ depending on time with $k_{p}(0)=0$.

The plan of the paper is the following. In Section 2, we establish the local-in-time existence of solutions for the system (1.1)-(1.10). We then obtain a priori estimates in Section 3 to prove Theorem 1.1. Finally Section 4 is devoted to the asymptotic analysis of the solutions in order to prove Theorem 1.2.

Notation. For any $a \in \mathbb{R}^{3}$, we set

$$
\widehat{\mathcal{B}}(a)=\left\{x \in \mathbb{R}^{3}|| x-a \mid<1\right\}, \quad \widehat{\mathcal{F}}(a)=\Omega \backslash \overline{\widehat{\mathcal{B}}(a)} .
$$

In particular,

$$
\mathcal{B}(t)=\widehat{\mathcal{B}}(h(t)), \quad \mathcal{F}(t)=\widehat{\mathcal{F}}(h(t)) .
$$

In this article, to shorten the notation, we write $H^{m}$ and $L^{2}$ instead of $H^{m}(\mathcal{F}(0))$ and $L^{2}(\mathcal{F}(0))$.

Assume $\mathfrak{X}$ is Banach space. We need to consider a particular norm for $H^{m}(0, T ; \mathfrak{X})$ if $m \in \mathbb{N}^{*}$ and if $T \in \mathbb{R}_{+}^{*}$.

$$
\|f\|_{H_{\infty}^{m}(0, T ; \mathfrak{X})}=\|f\|_{H^{m}(0, T ; \mathfrak{X})}+\|f\|_{W^{m-1, \infty}(0, T ; \mathfrak{X})} .
$$

Using the Sobolev embedding, this norm is equivalent to the usual one, but the corresponding constants depend on $T$ and that is the reason why we introduce such a notation.

Assume $\mathfrak{X}_{1}$ and $\mathfrak{X}_{2}$ are Banach spaces. We also introduce the following spaces

$$
H^{m}\left(0, T ; \mathfrak{X}_{1}, \mathfrak{X}_{2}\right)=L^{2}\left(0, T ; \mathfrak{X}_{1}\right) \cap H^{m}\left(0, T ; \mathfrak{X}_{2}\right) \quad(m \geqslant 1) .
$$

In the case $T \in \mathbb{R}_{+}^{*}$, we also need to introduce the following norm for the above space:

$$
\|f\|_{H_{\infty}^{1}\left(0, T ; H^{2}, L^{2}\right)}=\|f\|_{L^{2}\left(0, T ; H^{2}\right)}+\|f\|_{L^{\infty}\left(0, T ; H^{1}\right)}+\|f\|_{H^{1}\left(0, T ; L^{2}\right)},
$$




$$
\|f\|_{H_{\infty}^{2}\left(0, T ; H^{4}, L^{2}\right)}=\|f\|_{L^{2}\left(0, T ; H^{4}\right)}+\|f\|_{L^{\infty}\left(0, T ; H^{3}\right)}+\|f\|_{H^{1}\left(0, T ; H^{2}\right)}+\|f\|_{W^{1, \infty}\left(0, T ; H^{1}\right)}+\|f\|_{H^{2}\left(0, T ; L^{2}\right)} .
$$

Using interpolation results, we see again that the corresponding norm is equivalent to $H^{1}\left(0, T ; H^{2}\right)$ but the corresponding constants depend on $T$.

\section{Local in time EXISTENCE OF SOLUTIONS}

In order to prove Theorem 1.1, we first prove the existence and uniqueness of strong solutions of system (1.1)-(1.10) for small times. More precisely, we show in this section the following result:

Theorem 2.1. Let $h_{1} \in \Omega^{0}$ and $\bar{\rho}>0$. Assume $w$ is given by the feedback law (1.10) with $k_{d} \in \mathbb{R}$ and $k_{p} \in H_{l o c}^{1}([0, \infty))$. There exist $\delta_{0}, C_{*}, T_{*}>0$ such that for any

$$
h_{0} \in \Omega^{0}, \rho_{0} \in H^{3}, u_{0} \in H^{3}, \ell_{0}, \omega_{0} \in \mathbb{R}^{3},
$$

satisfying the compatibility conditions (1.14)-(1.16) with

$$
\left\|\left(\rho_{0}, u_{0}, \ell_{0}, \omega_{0}\right)\right\|_{\widehat{\mathcal{S}}_{0,0}}+\left|h_{1}-h_{0}\right| \leqslant \delta_{0}
$$

the system (1.1)-(1.9) admits a unique strong solution $(\rho, u, \ell, \omega) \in \widehat{\mathcal{S}}_{0, T_{*}}, h \in L^{\infty}\left(0, T_{*}\right)$ and

$$
\|(\rho, u, \ell, \omega)\|_{\widehat{\mathcal{S}}_{0, T_{*}}}+\left\|h_{1}-h\right\|_{L^{\infty}\left(0, T_{*}\right)} \leqslant C_{*}\left(\left\|\left(\rho_{0}, u_{0}, \ell_{0}, \omega_{0}\right)\right\|_{\widehat{\mathcal{S}}_{0,0}}+\left|h_{1}-h_{0}\right|\right) .
$$

2.1. Lagrangian change of variables. Firstly, we use a Lagrangian change of variables to rewrite the system (1.1)-(1.10) in a fixed spatial domain: let introduce the flow $X(t, \cdot): \overline{\mathcal{F}(0)} \rightarrow \overline{\mathcal{F}(t)}$ defined by

$$
\left\{\begin{array}{l}
\frac{\partial X}{\partial t}(t, y)=u(t, X(t, y)) \\
X(0, y)=y
\end{array}\right.
$$

Due to the boundary conditions, we have

$$
X(t, y)= \begin{cases}h(t)+Q(t)\left(y-h_{0}\right) & \text { if } y \in \partial \mathcal{B}(0), \\ y & \text { if } y \in \partial \Omega,\end{cases}
$$

where $Q(t) \in S O(3)$ is the rotation matrix associated to the angular velocity $\omega$ :

$$
Q^{\prime}=\mathbb{A}(\omega) Q, \quad Q(0)=\mathbb{I}_{3} .
$$

For any $\omega \in \mathbb{R}^{3}, \mathbb{A}(\omega)$ is the skew-symmetric matrix:

$$
\mathbb{A}(\omega)=\left(\begin{array}{ccc}
0 & -\omega_{3} & \omega_{2} \\
\omega_{3} & 0 & -\omega_{1} \\
-\omega_{2} & \omega_{1} & 0
\end{array}\right) .
$$

If $u$ is regular enough, $X$ is well-defined and $X(t, \cdot)$ is a $C^{1}$-diffeomorphism from $\overline{\mathcal{F}(0)}$ onto $\overline{\mathcal{F}(t)}$ for all $t \in(0, T)$. We denote by $Y(t, \cdot)$ the inverse of $X(t, \cdot)$ and we consider the following change of variables

$$
\begin{gathered}
\widetilde{u}(t, y)=Q(t)^{\top} u(t, X(t, y)), \quad \widetilde{\rho}(t, y)=\rho(t, X(t, y))-\bar{\rho}, \\
\widetilde{h}(t)=h(t)-h_{1}, \quad \widetilde{\ell}(t)=Q(t)^{\top} \ell(t), \quad \widetilde{\omega}(t)=Q(t)^{\top} \omega(t) .
\end{gathered}
$$

Note that now we have

$$
X(t, y)=y+\int_{0}^{t} Q(s) \widetilde{u}(s, y) d s, \quad \forall y \in \overline{\mathcal{F}(0)} .
$$


Under the change of variables (2.4)-(2.5), the system (1.1)-(1.9) is transformed as follows:

$$
\begin{aligned}
\frac{\partial \widetilde{\rho}}{\partial t}+\rho_{0} \operatorname{div} \widetilde{u} & =F_{1}(\widetilde{\rho}, \widetilde{u}, \widetilde{\ell}, \widetilde{\omega}, Q) \quad \text { in }(0, T) \times \mathcal{F}(0), \\
\frac{\partial \widetilde{u}}{\partial t}-\frac{\mu}{\rho_{0}} \Delta \widetilde{u}-\frac{\lambda+\mu}{\rho_{0}} \nabla(\operatorname{div} \widetilde{u}) & =F_{2}(\widetilde{\rho}, \widetilde{u}, \widetilde{\ell}, \widetilde{\omega}, Q) \quad \text { in } \quad(0, T) \times \mathcal{F}(0), \\
m \widetilde{\ell}^{\prime} & =F_{3}(\widetilde{\rho}, \widetilde{u}, \widetilde{h}, \widetilde{\ell}, \widetilde{\omega}, Q) \quad \text { in } \quad(0, T), \\
J^{\prime} & =F_{4}(\widetilde{\rho}, \widetilde{u}, \widetilde{\ell}, \widetilde{\omega}, Q) \quad \text { in } \quad(0, T), \\
\widetilde{h}^{\prime} & =Q \widetilde{\ell}, Q^{\prime}=Q \mathbb{A}(\widetilde{\omega}) \quad \text { in } \quad(0, T), \\
\widetilde{u} & =\widetilde{\ell}+\widetilde{\omega} \times\left(y-h_{0}\right) \quad \text { on } \quad(0, T) \times \partial \mathcal{B}(0), \\
\widetilde{u} & =0 \quad \text { in } \quad(0, T) \times \partial \Omega, \quad \\
\widetilde{\rho}(0, \cdot) & =\rho_{0}(\cdot)-\bar{\rho}, \quad \widetilde{u}(0, \cdot)=u_{0}(\cdot), \quad \text { in } \quad \mathcal{F}(0), \\
\widetilde{h}(0) & =h_{0}-h_{1}, \quad \widetilde{\ell}(0)=\ell_{0}, \quad \widetilde{\omega}(0)=\omega_{0}, \quad Q(0)=\mathbb{I}_{3} .
\end{aligned}
$$

In the above equations, $F_{1}, F_{2}, F_{3}, F_{4}$ are defined in the following way:

$$
F_{1}(\widetilde{\rho}, \widetilde{u}, \widetilde{\ell}, \widetilde{\omega}, Q)=-(\widetilde{\rho}+\bar{\rho}) \nabla \widetilde{u}:\left[((\nabla Y(X)) Q)^{\top}-\mathbb{I}_{3}\right]-\left(\widetilde{\rho}+\bar{\rho}-\rho_{0}\right) \operatorname{div} \widetilde{u},
$$

for $i=1,2,3$ :

$$
\begin{aligned}
& \left(F_{2}\right)_{i}(\widetilde{\rho}, \widetilde{u}, \widetilde{\ell}, \widetilde{\omega}, Q)=-(\widetilde{\omega} \times \widetilde{u})_{i}+\frac{\mu}{\widetilde{\rho}+\bar{\rho}} \sum_{p, l, m} \frac{\partial^{2} \widetilde{u}_{i}}{\partial y_{m} \partial y_{l}}\left(\frac{\partial Y_{m}}{\partial x_{p}}(X) \frac{\partial Y_{l}}{\partial x_{p}}(X)-\delta_{m p} \delta_{l p}\right) \\
& +\frac{\mu}{\widetilde{\rho}+\bar{\rho}} \sum_{p, l} \frac{\partial \widetilde{u}_{i}}{\partial y_{l}} \frac{\partial^{2} Y_{l}}{\partial x_{p}^{2}}(X)+\mu \Delta \widetilde{u}_{i}\left(\frac{\rho_{0}-(\widetilde{\rho}+\bar{\rho})}{\rho_{0}(\widetilde{\rho}+\bar{\rho})}\right)+\frac{\lambda+\mu}{\widetilde{\rho}+\bar{\rho}} \sum_{p, l} \frac{\partial \widetilde{u}_{p}}{\partial y_{l}} \frac{\partial^{2} Y_{l}}{\partial x_{p} \partial x_{i}}(X) \\
& +\frac{\lambda+\mu}{\widetilde{\rho}+\bar{\rho}} \sum_{p, l, m} \frac{\partial^{2} \widetilde{u}_{p}}{\partial y_{m} \partial y_{l}}\left(\frac{\partial Y_{m}}{\partial x_{p}}(X)-\delta_{m p}\right) \frac{\partial Y_{l}}{\partial x_{i}}(X)+\frac{\lambda+\mu}{\widetilde{\rho}+\bar{\rho}} \sum_{p, l} \frac{\partial^{2} \widetilde{u}_{p}}{\partial y_{p} \partial y_{l}}\left(\frac{\partial Y_{l}}{\partial x_{i}}(X)-\delta_{l i}\right) \\
& +(\lambda+\mu)[\nabla(\operatorname{div} \widetilde{u})]_{i}\left(\frac{\rho_{0}-(\widetilde{\rho}+\bar{\rho})}{\rho_{0}(\widetilde{\rho}+\bar{\rho})}\right)+a \gamma(\widetilde{\rho}+\bar{\rho})^{\gamma-2} \sum_{j, l} Q_{j i} \frac{\partial \widetilde{\rho}}{\partial y_{l}} \frac{\partial Y_{l}}{\partial x_{j}}(X), \\
& F_{3}(\widetilde{\rho}, \widetilde{u}, \widetilde{h}, \widetilde{\ell}, \widetilde{\omega}, Q)=-m(\widetilde{\omega} \times \widetilde{\ell})-\int_{\partial \mathcal{B}(0)}\left[\mu\left(Q \nabla \widetilde{u}(\nabla Y(X))+(Q \nabla \widetilde{u}(\nabla Y(X)))^{\top}\right)\right. \\
& \left.+\lambda\left(Q \nabla \widetilde{u}(\nabla Y(X)): \mathbb{I}_{3}\right)-a(\bar{\rho}+\widetilde{\rho})^{\gamma}\right] n d \Gamma-k_{p} Q^{\top} \widetilde{h}-k_{d} \widetilde{\ell}, \\
& F_{4}(\widetilde{\rho}, \widetilde{u}, \widetilde{\ell}, \widetilde{\omega}, Q)=-\int_{\partial \mathcal{B}(0)}\left(y-h_{0}\right) \times\left[\mu\left(Q \nabla \widetilde{u}(\nabla Y(X))+(Q \nabla \widetilde{u}(\nabla Y(X)))^{\top}\right)\right. \\
& \left.+\lambda(Q \nabla \widetilde{u}(\nabla Y(X)): \mathrm{Id})-a(\bar{\rho}+\widetilde{\rho})^{\gamma}\right] n d \Gamma .
\end{aligned}
$$

Here $n(y)=Q(t)^{\top} N(t, x)$ is the unit normal to $\partial \mathcal{B}(0)$ at the point $y \in \partial \mathcal{B}(0)$, directed to the interior of the ball. 
2.2. Analysis of a linear problem. In this section, we want to study the existence and regularity of the solution of the following linear system:

$$
\begin{aligned}
\frac{\partial \widetilde{\rho}}{\partial t}+\rho_{0} \operatorname{div} \widetilde{u} & =f_{1} \quad \text { in } \quad(0, T) \times \mathcal{F}(0), \\
\frac{\partial \widetilde{u}}{\partial t}-\frac{\mu}{\rho_{0}} \Delta \widetilde{u}-\frac{\lambda+\mu}{\rho_{0}} \nabla(\operatorname{div} \widetilde{u}) & =f_{2} \quad \text { in } \quad(0, T) \times \mathcal{F}(0), \\
m \widetilde{\ell^{\prime}} & =f_{3} \quad \text { in } \quad(0, T), \\
J \widetilde{\omega}^{\prime} & =f_{4} \quad \text { in } \quad(0, T), \\
\widetilde{u} & =\widetilde{\ell}+\widetilde{\omega} \times\left(y-h_{0}\right) \quad \text { on } \quad(0, T) \times \partial \mathcal{B}(0), \\
\widetilde{u} & =0 \quad \text { on } \quad(0, T) \times \partial \Omega, \\
\widetilde{u}(0, \cdot) & =u_{0}(\cdot) \quad \text { in } \quad \mathcal{F}(0), \\
\widetilde{\rho}(0, \cdot) & =\widetilde{\rho}_{0} \quad \text { in } \quad \mathcal{F}(0), \\
\widetilde{\ell}(0) & =\ell_{0}, \quad \widetilde{\omega}(0)=\omega_{0} .
\end{aligned}
$$

We introduce the following set for $T>0$ :

$$
\begin{array}{r}
\mathcal{S}_{T}=\left\{(\widetilde{\rho}, \widetilde{u}, \widetilde{\ell}, \widetilde{\omega}) \mid \widetilde{\rho} \in H^{1}\left(0, T ; H^{3}\right) \cap C^{1}\left([0, T] ; H^{2}\right) \cap H^{2}\left(0, T ; L^{2}\right), \widetilde{u} \in H^{2}\left(0, T ; H^{4}, L^{2}\right),\right. \\
\widetilde{\ell} \in H^{2}(0, T), \widetilde{\omega} \in H^{2}(0, T), \widetilde{u}=0 \text { on } \partial \Omega, \widetilde{u}=\widetilde{\ell}+\widetilde{\omega} \times\left(y-h_{0}\right) \text { on } \partial \mathcal{B}(0), \widetilde{\rho}(0)=\widetilde{\rho}_{0}, \\
\left.\widetilde{u}(0)=u_{0}, \widetilde{\ell}(0)=\ell_{0}, \widetilde{\omega}(0)=\omega_{0}\right\},
\end{array}
$$

equipped with the norm

$$
\begin{aligned}
\|(\widetilde{\rho}, \widetilde{u}, \widetilde{\ell}, \widetilde{\omega})\|_{\mathcal{S}_{T}}:=\|\widetilde{\rho}\|_{H_{\infty}^{1}\left(0, T ; H^{3}\right)}+\|\widetilde{\rho}\|_{W^{1, \infty}\left(0, T ; H^{2}\right)}+\|\widetilde{\rho}\|_{H^{2}\left(0, T ; L^{2}\right)}+\|\widetilde{u}\|_{H_{\infty}^{2}\left(0, T ; H^{4}, L^{2}\right)} \\
+\|\widetilde{\ell}\|_{H_{\infty}^{2}(0, T)}+\|\widetilde{\omega}\|_{H_{\infty}^{2}(0, T)} .
\end{aligned}
$$

We recall that the norms $\|\cdot\|_{H_{\infty}^{1}\left(0, T ; H^{3}\right)},\|\cdot\|_{H_{\infty}^{2}(0, T)}$ are defined in $(1.24)$ and $\|\cdot\|_{H_{\infty}^{2}\left(0, T ; H^{4}, L^{2}\right)}$ is defined in (1.26). The space $\mathcal{S}_{T}$ is similar to $\widehat{\mathcal{S}}_{T_{1}, T_{2}}$ defined by (1.12) except that here $\mathcal{F}(t)$ is replaced by $\mathcal{F}(0)$ and we add the boundary and initial conditions.

Since $\bar{\rho}>0$, there exists $\delta_{0}>0$ such that (2.2) implies

$$
\rho_{0} \geqslant \frac{\bar{\rho}}{2}>0
$$

In that case, the system (2.20)-(2.28) is well-posed:

Proposition 2.2. Let us assume $\bar{\rho}>0$, (2.2) with $\delta_{0}$ as above and

$$
\begin{gathered}
\left(\widetilde{\rho}_{0}, u_{0}, \ell_{0}, \omega_{0}\right) \in H^{3} \times H^{3} \times \mathbb{R}^{3} \times \mathbb{R}^{3}, \quad f_{1} \in L^{2}\left(0, T ; H^{3}\right) \cap C\left([0, T] ; H^{2}\right) \cap H^{1}\left(0, T ; L^{2}\right), \\
f_{2} \in H^{1}\left(0, T ; H^{2}, L^{2}\right), \quad f_{3} \in H^{1}(0, T), \quad f_{4} \in H^{1}(0, T)
\end{gathered}
$$

with

$$
\begin{gathered}
u_{0}=\ell_{0}+\omega_{0} \times\left(y-h_{0}\right) \text { for } y \in \partial \mathcal{B}(0), \quad u_{0}=0 \text { on } \partial \Omega, \\
f_{2}(0)+\frac{\mu}{\rho_{0}} \Delta u_{0}+\frac{\lambda+\mu}{\rho_{0}} \nabla\left(\operatorname{div} u_{0}\right)=0 \text { on } \partial \Omega, \\
f_{2}(0)+\frac{\mu}{\rho_{0}} \Delta u_{0}+\frac{\lambda+\mu}{\rho_{0}} \nabla\left(\operatorname{div} u_{0}\right)=m^{-1} f_{3}(0)+J^{-1} f_{4}(0) \times\left(y-h_{0}\right) \text { for } y \in \partial \mathcal{B}(0) .
\end{gathered}
$$


Then the system $(2.20)-(2.28)$ admits a unique solution $(\widetilde{\rho}, \widetilde{u}, \widetilde{\ell}, \widetilde{\omega}) \in \mathcal{S}_{T}$. Moreover, there exists $C_{L}>0$ (nondecreasing with respect to $T$ ) such that

$$
\begin{array}{r}
\|(\widetilde{\rho}, \widetilde{u}, \widetilde{\ell}, \widetilde{\omega})\|_{\mathcal{S}_{T}} \leqslant C_{L}\left(\left\|f_{1}\right\|_{L^{2}\left(0, T ; H^{3}\right)}+\left\|f_{1}\right\|_{L^{\infty}\left(0, T ; H^{2}\right)}+\left\|f_{1}\right\|_{H^{1}\left(0, T ; L^{2}\right)}+\left\|f_{2}\right\|_{H_{\infty}^{1}\left(0, T ; L^{2}, H^{2}\right)}\right. \\
\left.+\left\|f_{3}\right\|_{H_{\infty}^{1}(0, T)}+\left\|f_{4}\right\|_{H_{\infty}^{1}(0, T)}+\left\|\widetilde{\rho}_{0}\right\|_{H^{3}}+\left\|u_{0}\right\|_{H^{3}}+\left|\ell_{0}\right|+\left|\omega_{0}\right|\right) .
\end{array}
$$

Proof. We solve (2.20)-(2.28) like a cascade system: first, (2.22)-(2.23) admits a unique solution $(\widetilde{\ell}, \widetilde{\omega})$ with

$$
\|\widetilde{\ell}\|_{H_{\infty}^{2}(0, T)}+\|\widetilde{\omega}\|_{H_{\infty}^{2}(0, T)} \leqslant C\left(\left\|f_{3}\right\|_{H_{\infty}^{1}(0, T)}+\left\|f_{4}\right\|_{H_{\infty}^{1}(0, T)}+\left|\ell_{0}\right|+\left|\omega_{0}\right|\right) .
$$

Next, we solve equation (2.21) with the boundary and initial conditions (2.24)-(2.26). First we consider a lifting operator $\mathcal{R}$, such that for any $a, b \in \mathbb{R}^{3}, \mathcal{R}(a, b) \in C^{\infty}\left(\mathbb{R}^{3}\right)$ satisfies

$$
\mathcal{R}(a, b)= \begin{cases}a+b \times\left(y-h_{0}\right) & \text { on } \partial \mathcal{B}(0), \\ 0 & \text { on } \partial \Omega\end{cases}
$$

Then $\widetilde{v}=\widetilde{u}-\mathcal{R}(\widetilde{\ell}, \widetilde{\omega})$ satisfies

$$
\left\{\begin{aligned}
\frac{\partial \widetilde{v}}{\partial t}-\frac{\mu}{\rho_{0}} \Delta \widetilde{v}-\frac{\lambda+\mu}{\rho_{0}} \nabla(\operatorname{div} \widetilde{v}) & =F=f_{2}+\frac{\mu}{\rho_{0}} \Delta \mathcal{R}(\widetilde{\ell}, \widetilde{\omega})+\frac{\lambda+\mu}{\rho_{0}} \nabla(\operatorname{div} \mathcal{R}(\widetilde{\ell}, \widetilde{\omega}))-\mathcal{R}\left(\widetilde{\ell}^{\prime}, \widetilde{\omega}^{\prime}\right), \\
& \widetilde{v}=0 \text { on }(0, T) \times \partial \mathcal{F}(0), \quad \text { in } \mathcal{F}(0) .
\end{aligned}\right.
$$

By using a standard Galerkin method (see [8, Chapter 7, Theorem 1, p.354]) and by using the regularity result of Lamé operator (see, for instance, [4, Theorem 6.3-6, p.296]), under the condition that $\partial \mathcal{F}(0)$ is of class $C^{4}$, we can show the following result: if

$$
F \in L^{2}\left(0, T ; H^{2}\right) \cap H^{1}\left(0, T ; L^{2}\right), \quad \widetilde{v}_{0} \in H^{3} \cap H_{0}^{1},
$$

with the condition

$$
F(0, \cdot)+\frac{\mu}{\rho_{0}} \Delta \widetilde{v}_{0}+\frac{\lambda+\mu}{\rho_{0}} \nabla\left(\operatorname{div} \widetilde{v}_{0}\right)=0 \quad \text { on } \partial \mathcal{F}(0),
$$

then there exists a unique solution $\widetilde{v} \in H^{2}\left(0, T ; H^{4}, L^{2}\right)$ with the estimate

$$
\|\widetilde{v}\|_{H_{\infty}^{2}\left(0, T ; H^{4}, L^{2}\right)} \leqslant C\left(\|F\|_{H_{\infty}^{1}\left(0, T ; L^{2}, H^{2}\right)}+\|\widetilde{v}(0)\|_{H^{3}}\right) .
$$

We note that condition (2.35) is equivalent to (2.31) and (2.32). We can use the relation $\widetilde{u}=\widetilde{v}+\mathcal{R}(\widetilde{\ell}, \widetilde{\omega})$ and the above estimate of $\widetilde{v}$ to deduce the following estimate of $\widetilde{u}$ :

$$
\|\widetilde{u}\|_{H_{\infty}^{2}\left(0, T ; H^{4}, L^{2}\right)} \leqslant C\left(\left\|f_{2}\right\|_{H_{\infty}^{1}\left(0, T ; L^{2}, H^{2}\right)}+\left\|f_{3}\right\|_{H_{\infty}^{1}(0, T)}+\left\|f_{4}\right\|_{H_{\infty}^{1}(0, T)}+\left\|u_{0}\right\|_{H^{3}}+\left|\ell_{0}\right|+\left|\omega_{0}\right|\right) .
$$

Now, with the help of equation (2.20) satisfied by $\widetilde{\rho}$, we obtain

$$
\begin{aligned}
\|\widetilde{\rho}\|_{H_{\infty}^{1}\left(0, T ; H^{3}\right)}+\|\widetilde{\rho}\|_{W^{1, \infty}\left(0, T ; H^{2}\right)} & +\|\widetilde{\rho}\|_{H^{2}\left(0, T ; L^{2}\right)} \leqslant C\left(\left\|f_{1}\right\|_{L^{2}\left(0, T ; H^{3}\right)}+\left\|f_{1}\right\|_{L^{\infty}\left(0, T ; H^{2}\right)}+\left\|f_{1}\right\|_{H^{1}\left(0, T ; L^{2}\right)}\right. \\
& \left.+\|\widetilde{u}\|_{L^{2}\left(0, T ; H^{4}\right)}+\|\widetilde{u}\|_{L^{\infty}\left(0, T ; H^{3}\right)}+\|\widetilde{u}\|_{H^{1}\left(0, T ; H^{1}\right)}+\left\|\widetilde{\rho}_{0}\right\|_{H^{3}}\right) . \quad(2.37)
\end{aligned}
$$

Thus, we have proved the existence of solution in appropriate space for the system (2.20)-(2.28). Thanks to (2.34), (2.36) and (2.37), we have also obtained our required estimate (2.33). 
2.3. Estimates of the nonlinear terms. For $T>0$ and $R>0$, we define the following subset of $\mathcal{S}_{T}$ :

$$
\mathcal{S}_{T, R}=\left\{(\widetilde{\rho}, \widetilde{u}, \widetilde{\ell}, \widetilde{\omega}) \in \mathcal{S}_{T} \mid\|(\widetilde{\rho}, \widetilde{u}, \widetilde{\ell}, \widetilde{\omega})\|_{\mathcal{S}_{T}} \leqslant R\right\} .
$$

In what follows, $R$ is fixed and the constants that appear can depend on $R$.

Assume $(\widetilde{\rho}, \widetilde{u}, \widetilde{\ell}, \widetilde{\omega}) \in \mathcal{S}_{T, R}$. Then there exists a unique solution $(\widetilde{h}, Q) \in H^{3}(0, T)$ of the following equations

$$
\left\{\begin{array}{l}
\widetilde{h}^{\prime}=Q \widetilde{\ell} \quad \text { in } \quad(0, T) \\
Q^{\prime}=Q \mathbb{A}(\widetilde{\omega}) \quad \text { in } \quad(0, T) \\
Q(0)=\mathbb{I}_{3}, \quad \widetilde{h}(0)=h_{0}-h_{1}
\end{array}\right.
$$

and we can then define $X$ by (2.6). From (2.38), there exists $C=C(R)>0$ such that

$$
\begin{gathered}
\|Q\|_{H^{3}(0, T)} \leqslant C, \quad\left\|Q-\mathbb{I}_{3}\right\|_{L^{\infty}(0, T)} \leqslant C T, \\
\|\widetilde{h}\|_{L^{\infty}(0, T)} \leqslant\left|h_{0}-h_{1}\right|+C T^{1 / 2} .
\end{gathered}
$$

In particular, taking $\delta_{0}$ small enough in $(2.2)$, there exists $T_{1}=T_{1}\left(R, \delta_{0}, \operatorname{dist}\left(h_{1}, \partial \Omega\right)\right)>0$ and $c_{1}>0$ such that

$$
\operatorname{dist}\left(\widehat{\mathcal{B}}\left(\widetilde{h}(t)+h_{1}\right), \partial \Omega\right) \geqslant c_{1}>0 \quad \forall t \in\left[0, T_{1}\right] .
$$

From now on, we assume $T \leqslant T_{1}$ and the constants may depend on $T_{1}$.

Combining (2.6) and (2.38), we also deduce

$$
\left\|\nabla X-\mathbb{I}_{3}\right\|_{L^{\infty}\left(0, T ; H^{3}\right)} \leqslant C T^{1 / 2} .
$$

In particular, using the embedding $H^{3}(\mathcal{F}(0)) \hookrightarrow W^{1, \infty}(\mathcal{F}(0))$ and $(2.41)$, there exists $T_{2} \leqslant T_{1}$ such that $X: \mathcal{F}(0) \rightarrow \widehat{\mathcal{F}}\left(\widetilde{h}(t)+h_{1}\right)$ is invertible and its inverse is denoted by $Y$.

In the same spirit, using the initial condition on $\widetilde{\rho}$ (see (2.29)), we have

$$
\left\|\widetilde{\rho}+\bar{\rho}-\rho_{0}\right\|_{L^{\infty}\left(0, T ; H^{3}\right)} \leqslant T^{1 / 2} R .
$$

Using the embedding $H^{3}(\mathcal{F}(0)) \hookrightarrow L^{\infty}(\mathcal{F}(0))$ and $(2.2)$ with $\delta_{0}$ small enough, there exists $T_{3} \leqslant T_{2}$ such that

$$
\frac{\bar{\rho}}{2} \leqslant \widetilde{\rho}+\bar{\rho} \leqslant \frac{3 \bar{\rho}}{2} .
$$

In particular, combining this with (2.38), for any $\alpha \in \mathbb{R}$,

$$
\left\|(\widetilde{\rho}+\bar{\rho})^{\alpha}\right\|_{L^{\infty}\left(0, T ; H^{3}\right)} \leqslant C, \quad\left\|\int_{\partial \mathcal{B}(0)}(\widetilde{\rho}+\bar{\rho})^{\gamma} n d \Gamma\right\|_{H^{1}(0, T)} \leqslant C T^{1 / 2} .
$$

From the above construction and assuming $T \leqslant T_{3}$, we can define the terms $F_{1}, F_{2}, F_{3}, F_{4}$ by (2.16)-(2.19). To estimate these terms, we first give some estimates of $X$ and $Y$ :

Lemma 2.3. Assume $(\widetilde{\rho}, \widetilde{u}, \widetilde{\ell}, \widetilde{\omega}) \in \mathcal{S}_{T, R}$. There exists a positive constant $C$ depending only on $R$, $\mathcal{F}(0)$ such that, for all $0<T \leqslant T_{3}$,

$$
\left\|\frac{\partial^{2} Y_{l}}{\partial x_{p} \partial x_{i}}(X)\right\|_{L^{\infty}\left(0, T ; H^{2}\right)}+\left\|\frac{\partial}{\partial t}(\nabla Y(X))\right\|_{L^{\infty}\left(0, T ; H^{2}\right)}+\left\|\frac{\partial}{\partial t}\left(\frac{\partial^{2} Y_{l}}{\partial x_{p} \partial x_{i}}(X)\right)\right\|_{L^{\infty}\left(0, T ; H^{1}\right)} \leqslant C .
$$


Proof. From (2.42) and the fact that $L^{\infty}\left(0, T ; H^{3}\right)$ is an algebra, we deduce (2.46). This yields in particular that

Writing

$$
\|\nabla Y(X)\|_{L^{\infty}\left(0, T ; H^{3}\right)} \leqslant C
$$

$$
\frac{\partial}{\partial y_{m}}\left(\frac{\partial Y_{l}}{\partial x_{i}}(X)\right)=\sum_{p} \frac{\partial^{2} Y_{l}}{\partial x_{p} \partial x_{i}}(X) \frac{\partial X_{p}}{\partial y_{m}}
$$

and using (2.48), we deduce the estimate on $\frac{\partial^{2} Y_{l}}{\partial x_{p} \partial x_{i}}(X)$.

From the expression (2.6), we have $\frac{\partial}{\partial t}(\nabla X(t, \cdot))=Q(t) \nabla \widetilde{u}(t, \cdot)$, and using

$$
\frac{\partial}{\partial t}(\nabla Y(X))=-\nabla Y(X) \frac{\partial}{\partial t}(\nabla X) \nabla Y(X)
$$

we obtain the estimate of the second term in (2.47).

Finally, we write

$$
\frac{\partial}{\partial y_{m}}\left[\frac{\partial}{\partial t}\left(\frac{\partial Y_{l}}{\partial x_{i}}(X)\right)\right]=\sum_{p} \frac{\partial}{\partial t}\left(\frac{\partial^{2} Y_{l}}{\partial x_{p} \partial x_{i}}(X)\right) \frac{\partial X_{p}}{\partial y_{m}}+\sum_{p} \frac{\partial^{2} Y_{l}}{\partial x_{p} \partial x_{i}}(X) \frac{\partial}{\partial t}\left(\frac{\partial X_{p}}{\partial y_{m}}\right)
$$

and from the previous estimate, we have

$$
\left\|\frac{\partial}{\partial y_{m}}\left[\frac{\partial}{\partial t}\left(\frac{\partial Y_{l}}{\partial x_{i}}(X)\right)\right]\right\|_{L^{\infty}\left(0, T ; H^{1}\right)}+\left\|\sum_{p} \frac{\partial^{2} Y_{l}}{\partial x_{p} \partial x_{i}}(X) \frac{\partial}{\partial t}\left(\frac{\partial X_{p}}{\partial y_{m}}\right)\right\|_{L^{\infty}\left(0, T ; H^{1}\right)} \leqslant C .
$$

Thus, using (2.48), we deduce the estimate of the last term in (2.47).

Next we give some properties on $F_{1}, F_{2}, F_{3}, F_{4}$.

Proposition 2.4. There exist $\alpha>0$ and a positive constant $C$ depending on $R, k_{p}, k_{d}, \bar{\rho}$ and the other physical parameters, and on $\mathcal{F}(0)$ such that, for all $0<T \leqslant T_{3}$, for all

$$
\begin{gathered}
(\widetilde{\rho}, \widetilde{u}, \widetilde{\ell}, \widetilde{\omega}),\left(\widetilde{\rho}^{1}, \widetilde{u}^{1}, \widetilde{\ell}^{1}, \widetilde{\omega}^{1}\right),\left(\widetilde{\rho}^{2}, \widetilde{u}^{2}, \widetilde{\ell}^{2}, \widetilde{\omega}^{2}\right) \in \mathcal{S}_{T, R}, \\
\left\|F_{1}(\widetilde{\rho}, \widetilde{u}, \widetilde{\ell}, \widetilde{\omega}, Q)\right\|_{L^{2}\left(0, T ; H^{3}\right) \cap L^{\infty}\left(0, T ; H^{2}\right) \cap H^{1}\left(0, T ; L^{2}\right)} \leqslant C T^{\alpha}, \\
\left\|F_{2}(\widetilde{\rho}, \widetilde{u}, \widetilde{\ell}, \widetilde{\omega}, Q)\right\|_{H_{\infty}^{1}\left(0, T ; L^{2}, H^{2}\right)} \leqslant C\left(T^{\alpha}+\left\|\omega_{0} \times u_{0}\right\|_{H^{1}}+\left\|a \gamma \rho_{0}^{\gamma-2} \nabla \rho_{0}\right\|_{H^{1}}\right), \\
\left\|F_{3}(\widetilde{\rho}, \widetilde{u}, \widetilde{h}, \widetilde{\ell}, \widetilde{\omega}, Q)\right\|_{H_{\infty}^{1}(0, T)} \leqslant C\left(T^{\alpha}+\left|\omega_{0} \times \ell_{0}\right|+\left|\ell_{0}\right|+\left\|\rho_{0}-\bar{\rho}\right\|_{H^{1}}+\left\|u_{0}\right\|_{H^{3}}\right), \\
\left\|F_{4}(\widetilde{\rho}, \widetilde{u}, \widetilde{\ell}, \widetilde{\omega}, Q)\right\|_{H_{\infty}^{1}(0, T)} \leqslant C T^{\alpha},
\end{gathered}
$$

and

$$
\begin{aligned}
& \left\|F_{1}\left(\widetilde{\rho}^{1}, \widetilde{u}^{1}, \widetilde{\ell}^{1}, \widetilde{\omega}^{1}, Q^{1}\right)-F_{1}\left(\widetilde{\rho}^{2}, \widetilde{u}^{2}, \widetilde{\ell}^{2}, \widetilde{\omega}^{2}, Q^{2}\right)\right\|_{L^{2}\left(0, T ; H^{3}\right) \cap L^{\infty}\left(0, T ; H^{2}\right) \cap H^{1}\left(0, T ; L^{2}\right)} \\
& \leqslant C T^{\alpha}\left\|\left(\widetilde{\rho}^{1}, \widetilde{u}^{1}, \widetilde{\ell}^{1}, \widetilde{\omega}^{1}\right)-\left(\widetilde{\rho}^{2}, \widetilde{u}^{2}, \widetilde{\ell}^{2}, \widetilde{\omega}^{2}\right)\right\|_{\mathcal{S}_{T},}, \\
& \left\|F_{2}\left(\widetilde{\rho}^{1}, \widetilde{u}^{1}, \widetilde{\ell}^{1}, \widetilde{\omega}^{1}, Q^{1}\right)-F_{2}\left(\widetilde{\rho}^{2}, \widetilde{u}^{2}, \widetilde{\ell}^{2}, \widetilde{\omega}^{2}, Q^{2}\right)\right\|_{H_{\infty}^{1}\left(0, T ; L^{2}, H^{2}\right)} \leqslant C T^{\alpha}\left\|\left(\widetilde{\rho}^{1}, \widetilde{u}^{1}, \widetilde{\ell}^{1}, \widetilde{\omega}^{1}\right)-\left(\widetilde{\rho}^{2}, \widetilde{u}^{2}, \widetilde{\ell}^{2}, \widetilde{\omega}^{2}\right)\right\|_{\mathcal{S}_{T}}, \\
& \left\|F_{3}\left(\widetilde{\rho}^{1}, \widetilde{u}^{1}, \widetilde{h}^{1}, \widetilde{\ell}^{1}, \widetilde{\omega}^{1}, Q^{1}\right)-F_{3}\left(\widetilde{\rho}^{2}, \widetilde{u}^{2}, \widetilde{h}^{2}, \widetilde{\ell}^{2}, \widetilde{\omega}^{2}, Q^{2}\right)\right\|_{H_{\infty}^{1}(0, T)} \leqslant C T^{\alpha}\left\|\left(\widetilde{\rho}^{1}, \widetilde{u}^{1}, \widetilde{\ell}^{1}, \widetilde{\omega}^{1}\right)-\left(\widetilde{\rho}^{2}, \widetilde{u}^{2}, \widetilde{\ell}^{2}, \widetilde{\omega}^{2}\right)\right\|_{\mathcal{S}_{T}}, \\
& \qquad\left\|F_{4}\left(\widetilde{\rho}^{1}, \widetilde{u}^{1}, \widetilde{\ell}^{1}, \widetilde{\omega}^{1}, Q^{1}\right)-F_{4}\left(\widetilde{\rho}^{2}, \widetilde{u}^{2}, \widetilde{\ell}^{2}, \widetilde{\omega}^{2}, Q^{2}\right)\right\|_{H_{\infty}^{1}(0, T)} \leqslant C T^{\alpha}\left\|\left(\widetilde{\rho}^{1}, \widetilde{u}^{1}, \widetilde{\ell}^{1}, \widetilde{\omega}^{1}\right)-\left(\widetilde{\rho}^{2}, \widetilde{u}^{2}, \widetilde{\ell}^{2}, \widetilde{\omega}^{2}\right)\right\|_{\mathcal{S}_{T}} . \\
& \text { where } Q, Q^{1}, Q^{2}, \widetilde{h}, \widetilde{h}^{1}, \widetilde{h}^{2} \in H^{3}(0, T) \text { are given by }(2.39) .
\end{aligned}
$$


Proof. Using the definition (2.16) of $F_{1},(2.43),(2.29),(2.38),(2.46)$ we have the following estimates

$$
\begin{aligned}
& \left\|F_{1}\right\|_{L^{2}\left(0, T ; H^{3}\right)} \leqslant C\|(\widetilde{\rho}+\bar{\rho})\|_{L^{\infty}\left(0, T ; H^{3}\right)}\|\nabla \widetilde{u}\|_{L^{2}\left(0, T ; H^{3}\right)}\left\|((\nabla Y) Q)^{\top}-\mathbb{I}_{3}\right\|_{L^{\infty}\left(0, T ; H^{3}\right)} \\
& +C\left\|\left(\widetilde{\rho}+\bar{\rho}-\rho_{0}\right)\right\|_{L^{\infty}\left(0, T ; H^{3}\right)}\|\operatorname{div} \widetilde{u}\|_{L^{2}\left(0, T ; H^{3}\right)} \leqslant C T^{\alpha}, \\
& \left\|\frac{\partial F_{1}}{\partial t}\right\|_{L^{2}\left(0, T ; L^{2}\right)} \leqslant C\|(\widetilde{\rho}+\bar{\rho})\|_{L^{\infty}\left(0, T ; H^{3}\right)}\left\{\left\|\nabla \frac{\partial \widetilde{u}}{\partial t}\right\|_{L^{2}\left(0, T ; L^{2}\right)}\left\|((\nabla Y) Q)^{\top}-\mathbb{I}_{3}\right\|_{L^{\infty}\left(0, T ; H^{3}\right)}\right. \\
& \left.+\|\nabla \widetilde{u}\|_{L^{2}\left(0, T ; H^{3}\right)}\left\|\frac{\partial}{\partial t}((\nabla Y(X)) Q)^{\top}\right\|_{L^{\infty}\left(0, T ; H^{2}\right)}\right\} \\
& +C T^{1 / 2}\left\|\frac{\partial \widetilde{\rho}}{\partial t}\right\|_{L^{\infty}\left(0, T ; H^{2}\right)}\|\nabla \widetilde{u}\|_{L^{\infty}\left(0, T ; H^{2}\right)}\left\|((\nabla Y(X)) Q)^{\top}\right\|_{L^{\infty}\left(0, T ; H^{3}\right)} \\
& +C\left\|\widetilde{\rho}+\bar{\rho}-\rho_{0}\right\|_{L^{\infty}\left(0, T ; H^{3}\right)}\left\|\operatorname{div} \frac{\partial \widetilde{u}}{\partial t}\right\|_{L^{2}\left(0, T ; H^{1}\right)} \leqslant C T^{\alpha} \\
& \left\|F_{1}\right\|_{L^{\infty}\left(0, T ; H^{2}\right)} \leqslant C\|\widetilde{\rho}+\bar{\rho}\|_{L^{\infty}\left(0, T ; H^{3}\right)}\|\nabla \widetilde{u}\|_{L^{\infty}\left(0, T ; H^{2}\right)}\left\|((\nabla Y) Q)^{\top}-\mathbb{I}_{3}\right\|_{L^{\infty}\left(0, T ; H^{3}\right)} \\
& +C\left\|\widetilde{\rho}+\bar{\rho}-\rho_{0}\right\|_{L^{\infty}\left(0, T ; H^{3}\right)}\|\operatorname{div} \widetilde{u}\|_{L^{\infty}\left(0, T ; H^{2}\right)} \leqslant C T^{1 / 2} .
\end{aligned}
$$

Let us now estimate the $L^{2}\left(0, T ; H^{2}\right)$ norm of $F_{2}$. Here we only estimate some terms in $(2.17)$, the other terms can be estimated similarly. Using (2.45), (2.29), (2.38), (2.46), (2.47),

$$
\begin{gathered}
\left\|\frac{1}{\widetilde{\rho}+\bar{\rho}} \frac{\partial^{2} \widetilde{u}_{i}}{\partial y_{m} \partial y_{l}}\left(\frac{\partial Y_{m}}{\partial x_{p}}(X) \frac{\partial Y_{l}}{\partial x_{p}}(X)-\delta_{m p} \delta_{l p}\right)\right\|_{L^{2}\left(0, T ; H^{2}\right)}\left\|\frac{\partial^{2} \widetilde{u}_{i}}{\partial y_{m} \partial y_{l}}\right\|_{L^{2}\left(0, T ; H^{2}\right)}\left\|\frac{\partial Y_{m}}{\partial x_{p}}(X) \frac{\partial Y_{l}}{\partial x_{p}}(X)-\delta_{m p} \delta_{l p}\right\|_{L^{\infty}\left(0, T ; H^{2}\right)} \\
\leqslant C\left\|\frac{1}{\widetilde{\rho}+\bar{\rho}}\right\|_{L^{\infty}\left(0, T ; H^{3}\right)} \leqslant T^{\alpha}, \\
\left\|\frac{1}{\widetilde{\rho}+\bar{\rho}} \frac{\partial \widetilde{u}_{i}}{\partial y_{l}} \frac{\partial^{2} Y_{l}}{\partial x_{p}^{2}}(X)\right\|_{L^{2}\left(0, T ; H^{2}\right)} \leqslant C T^{1 / 2}\left\|\frac{1}{\widetilde{\rho}+\bar{\rho}}\right\|_{L^{\infty}\left(0, T ; H^{3}\right)}\left\|\frac{\partial \widetilde{u}_{i}}{\partial y_{l}}\right\|_{L^{\infty}\left(0, T ; H^{2}\right)}\left\|\frac{\partial^{2} Y_{l}}{\partial x_{p}^{2}}(X)\right\|_{L^{\infty}\left(0, T ; H^{2}\right)} \\
\| C T^{\alpha}, \\
\left\|(\widetilde{\rho}+\bar{\rho})^{\gamma-2} \frac{\partial \widetilde{\rho}}{\partial y_{l}} \frac{\partial Y_{l}}{\partial x_{j}}(X)\right\|_{L^{2}\left(0, T ; H^{2}\right)}\left\|\frac{\partial Y_{l}}{\partial x_{j}}(X)\right\|_{L^{\infty}\left(0, T ; H^{2}\right)} \leqslant C T^{\alpha} . \\
\leqslant C T^{1 / 2}\left\|(\widetilde{\rho}+\bar{\rho})^{\gamma-2}\right\|_{L^{\infty}\left(0, T ; H^{2}\right)}\left\|\frac{\partial \widetilde{\rho}}{\partial y_{l}}\right\|_{L^{\infty}\left(0, T ; H^{2}\right)}
\end{gathered}
$$

For the estimate of the $H^{1}\left(0, T ; L^{2}\right)$ norm of $F_{2}$, we also only give the estimates the $L^{2}\left(0, T ; L^{2}\right)$ norm of some terms of the time derivative $F_{2}$. Again, the other terms can be estimated similarly. 
First, we write

$$
\begin{gathered}
\frac{\partial}{\partial t}\left[\frac{1}{\widetilde{\rho}+\bar{\rho}} \frac{\partial^{2} \widetilde{u}_{i}}{\partial y_{m} \partial y_{l}}\left(\frac{\partial Y_{m}}{\partial x_{p}}(X) \frac{\partial Y_{l}}{\partial x_{p}}(X)-\delta_{m p} \delta_{l p}\right)\right] \\
=-\frac{1}{(\widetilde{\rho}+\bar{\rho})^{2}} \frac{\partial \widetilde{\rho}}{\partial t} \frac{\partial^{2} \widetilde{u}_{i}}{\partial y_{m} \partial y_{l}}\left(\frac{\partial Y_{m}}{\partial x_{p}}(X) \frac{\partial Y_{l}}{\partial x_{p}}(X)-\delta_{m p} \delta_{l p}\right) \\
+\left(\frac{1}{\widetilde{\rho}+\bar{\rho}}\right) \frac{\partial^{3} \widetilde{u}_{i}}{\partial t \partial y_{m} \partial y_{l}}\left(\frac{\partial Y_{m}}{\partial x_{p}}(X) \frac{\partial Y_{l}}{\partial x_{p}}(X)-\delta_{m p} \delta_{l p}\right)+\left(\frac{1}{\widetilde{\rho}+\bar{\rho}}\right) \frac{\partial^{2} \widetilde{u}_{i}}{\partial y_{m} \partial y_{l}} \frac{\partial}{\partial t}\left(\frac{\partial Y_{m}}{\partial x_{p}}(X) \frac{\partial Y_{l}}{\partial x_{p}}(X)\right) \\
\frac{\partial}{\partial t}\left[\frac{1}{\widetilde{\rho}+\bar{\rho}} \frac{\partial \widetilde{u}_{i}}{\partial y_{l}} \frac{\partial^{2} Y_{l}}{\partial x_{p}^{2}}(X)\right]=-\frac{1}{(\widetilde{\rho}+\bar{\rho})^{2}} \frac{\partial \widetilde{\rho}}{\partial t} \frac{\partial \widetilde{u}_{i}}{\partial y_{l}} \frac{\partial^{2} Y_{l}}{\partial x_{p}^{2}}(X)+\frac{1}{\widetilde{\rho}+\bar{\rho}} \frac{\partial^{2} \widetilde{u}_{i}}{\partial t \partial y_{l}} \frac{\partial^{2} Y_{l}}{\partial x_{p}^{2}}(X) \\
+\frac{1}{\widetilde{\rho}+\bar{\rho}} \frac{\partial \widetilde{u}_{i}}{\partial y_{l}} \frac{\partial}{\partial t}\left(\frac{\partial^{2} Y_{l}}{\partial x_{p}^{2}}(X)\right)
\end{gathered}
$$

Using (2.45), (2.29), (2.38), (2.46), (2.47), we deduce that the above terms is estimated in $L^{2}\left(0, T ; L^{2}\right)$ by $C T^{\alpha}$.

Finally, to obtain the $L^{\infty}\left(0, T ; H^{1}\right)$ estimate of the term $F_{2}$, we use the following inequality [15, Lemma 4.2]:

$$
\sup _{t \in(0, T)}\left\|F_{2}(t)\right\|_{H^{1}} \leqslant C\left(\left\|F_{2}\right\|_{L^{2}\left(0, T ; H^{2}\right)}+\left\|F_{2}\right\|_{H^{1}\left(0, T ; L^{2}\right)}+\left\|F_{2}(0)\right\|_{H^{1}}\right)
$$

and since

$$
\left\|F_{2}(0)\right\|_{H^{1}} \leqslant\left\|\omega_{0} \times u_{0}\right\|_{H^{1}}+\left\|a \gamma \rho_{0}^{\gamma-2} \nabla \rho_{0}\right\|_{H^{1}},
$$

we deduce the result for $F_{2}$.

It remains to estimate $F_{3}$ and $F_{4}$. We only consider $F_{3}$, the analysis for $F_{4}$ is the same. From (2.18), we can see that the time derivative of $F_{3}$ involves the following terms (and similar ones)

$$
\begin{array}{r}
(\widetilde{\omega} \times \widetilde{\ell})^{\prime}, \quad\left(k_{p} Q^{\top} \widetilde{h}\right)^{\prime}, \quad k_{d} \widetilde{\ell^{\prime}}, \quad \int_{\partial \mathcal{B}(0)}\left(Q^{\prime} \nabla \widetilde{u} \nabla Y(X)+Q \nabla \frac{\partial \widetilde{u}}{\partial t} \nabla Y(X)+Q \nabla \widetilde{u} \frac{\partial}{\partial t} \nabla Y(X)\right) n d \Gamma \\
-a \gamma \int_{\partial \mathcal{B}(0)}(\bar{\rho}+\widetilde{\rho})^{\gamma-1} \frac{\partial \widetilde{\rho}}{\partial t} n d \Gamma .
\end{array}
$$

Almost all the terms can be estimated in a direct way in $L^{2}(0, T)$ by using $(2.40),(2.45),(2.29),(2.38)$, (2.46). We have nevertheless to take care of

$$
\int_{\partial \mathcal{B}(0)} Q \nabla \frac{\partial \widetilde{u}}{\partial t} \nabla Y(X) n d \Gamma
$$

For this term, we use standard interpolation result (see, for instance, [2, Lemma A.5]) to obtain

$$
\left\|\nabla \frac{\partial \widetilde{u}}{\partial t}\right\|_{L^{8 / 3}\left(0, T ; H^{1 / 4}\right)} \leqslant C\left\|\nabla \frac{\partial \widetilde{u}}{\partial t}\right\|_{L^{\infty}\left(0, T ; L^{2}\right)}^{1 / 4}\left\|\nabla \frac{\partial \widetilde{u}}{\partial t}\right\|_{L^{2}\left(0, T ; H^{1}\right)}^{3 / 4},
$$

where $C$ is independent of $T$. Using a trace result and (2.29), (2.38), we deduce an estimate of $F_{3}^{\prime}$ in $L^{2}(0, T)$ of the form $C T^{\alpha}$. To end the estimate of $F_{3}$, we use that

$$
\left\|F_{3}\right\|_{L^{\infty}(0, T)} \leqslant\left|F_{3}(0)\right|+T^{1 / 2}\left\|F_{3}\right\|_{H^{1}(0, T)} .
$$


We have the following estimate:

Thus,

$$
\left|\int_{\partial \mathcal{B}(0)}(\bar{\rho}+\widetilde{\rho}(0))^{\gamma} n d \Gamma\right|=\left|\int_{\partial \mathcal{B}(0)} \rho_{0}^{\gamma} n d \Gamma\right|=\left|\int_{\partial \mathcal{B}(0)}\left(\rho_{0}^{\gamma}-\bar{\rho}^{\gamma}\right) n d \Gamma\right| \leqslant C \int_{\partial \mathcal{B}(0)}\left|\rho_{0}-\bar{\rho}\right| d \Gamma .
$$

$$
\left|F_{3}(0)\right| \leqslant C\left(\left|\omega_{0} \times \ell_{0}\right|+\left|\ell_{0}\right|+\left\|\rho_{0}-\bar{\rho}\right\|_{H^{1}}+\left\|u_{0}\right\|_{H^{3}}\right) .
$$

The estimates for the differences can be done in a similar way and we thus skip the corresponding proof.

\subsection{Proof of Theorem 2.1.}

Proof. We are going to establish the local in time existence of (2.7)-(2.19). In order to do this we use a fixed-point argument.

Assume $\bar{\rho}>0, \delta_{0}$ satisfying the smallness assumptions introduced in the above section and let us consider $\left(\rho_{0}, u_{0}, h_{0}, \ell_{0}, \omega_{0}\right)$ satisfying $(2.1),(2.2)$. Recall that from $(2.44)$, we have $\frac{\bar{\rho}}{2} \leqslant \rho_{0} \leqslant \frac{3 \bar{\rho}}{2}$ and thus, using Sobolev embeddings, there exists $C_{1}>0$ depending on $\bar{\rho}, \delta_{0}$ and the geometry such that

$$
C\left(\left\|\omega_{0} \times u_{0}\right\|_{H^{1}}+\left\|a \gamma \rho_{0}^{\gamma-2} \nabla \rho_{0}\right\|_{H^{1}}+\left|\omega_{0} \times \ell_{0}\right|+\left|\ell_{0}\right|+\left\|\rho_{0}-\bar{\rho}\right\|_{H^{1}}+\left\|u_{0}\right\|_{H^{3}}\right) \leqslant C_{1} \widetilde{\delta}_{0}
$$

where $C$ is the constant appearing in Proposition 2.4 and where we have set

$$
\widetilde{\delta}_{0}=\left\|\rho_{0}-\bar{\rho}\right\|_{H^{3}}+\left\|u_{0}\right\|_{H^{3}}+\left|h_{1}-h_{0}\right|+\left|\ell_{0}\right|+\left|\omega_{0}\right| \leqslant \delta_{0} .
$$

We now fix $R>0$ as

$$
R=2 C_{L} C_{1} \widetilde{\delta}_{0}
$$

where $C_{L}$ is the continuity constant in estimate (2.33). We take $T \leqslant T_{3}$, where $T_{3}=T_{3}(R)$ is the time obtained in the above section.

Let us define the following mapping

$$
\begin{aligned}
\mathcal{N}: & \mathcal{S}_{T, R} \rightarrow \mathcal{S}_{T, R} \\
& (\widetilde{\rho}, \widetilde{u}, \widetilde{\ell}, \widetilde{\omega}) \mapsto(\widehat{\rho}, \widehat{u}, \widehat{\ell}, \widehat{\omega}) .
\end{aligned}
$$

For $(\widetilde{\rho}, \widetilde{u}, \widetilde{\ell}, \widetilde{\omega}) \in \mathcal{S}_{T, R}$, we define $X$ by $(2.6), \widetilde{h}$ and $Q$ by (2.39) and $F_{1}, F_{2}, F_{3}, F_{4}$ by (2.16)-(2.19). Then $(\widehat{\rho}, \widehat{u}, \widehat{\ell}, \widehat{\omega})$ is the solution of

$$
\begin{gathered}
\frac{\partial \widehat{\rho}}{\partial t}+\rho_{0} \operatorname{div} \widehat{u}=F_{1}(\widetilde{\rho}, \widetilde{u}, \widetilde{\ell}, \widetilde{\omega}, Q) \quad \text { in }(0, T) \times \mathcal{F}(0), \\
\frac{\partial \widehat{u}}{\partial t}-\frac{\mu}{\rho_{0}} \Delta \widehat{u}-\frac{\lambda+\mu}{\rho_{0}} \nabla(\operatorname{div} \widehat{u})=F_{2}(\widetilde{\rho}, \widetilde{u}, \widetilde{\ell}, \widetilde{\omega}, Q) \quad \text { in } \quad(0, T) \times \mathcal{F}(0), \\
m \widehat{\ell}^{\prime}=F_{3}(\widetilde{\rho}, \widetilde{u}, \widetilde{h}, \widetilde{\ell}, \widetilde{\omega}, Q) \quad \text { in } \quad(0, T), \\
J \widehat{\omega}^{\prime}=F_{4}(\widetilde{\rho}, \widetilde{u}, \widetilde{\ell}, \widetilde{\omega}, Q) \quad \text { in } \quad(0, T), \\
\widehat{u}=\widehat{\ell}+\widehat{\omega} \times\left(y-h_{0}\right) \quad \text { on } \quad(0, T) \times \partial \mathcal{B}(0), \\
\widehat{u}=0 \quad \text { in } \quad(0, T) \times \partial \Omega . \\
\widehat{\rho}(0, \cdot)=\rho_{0}(\cdot)-\bar{\rho}, \quad \widehat{u}(0, \cdot)=u_{0}(\cdot) \quad \text { in } \quad \mathcal{F}(0), \\
\widehat{\ell}(0)=\ell_{0}, \quad \widehat{\omega}(0)=\omega_{0} .
\end{gathered}
$$


In order to show that $\mathcal{N}$ is well-defined, we apply Proposition 2.2 to the above system. First we note that (1.14)-(1.16) yield the compatibility conditions (2.30)-(2.32). More precisely, the first condition is exactly condition (1.14). Using the expression of $F_{2}$ in (2.17), we have

$$
\left[F_{2}(\widetilde{\rho}, \widetilde{u}, \widetilde{\ell}, \widetilde{\omega}, Q)\right](0, \cdot)=-\omega_{0} \times u_{0}+\frac{1}{\rho_{0}} \nabla p_{0},
$$

where $p_{0}=a \rho_{0}^{\gamma}$. Thus, (1.15) yields the second condition.

On the other hand, using the expressions of $F_{3}$ and $F_{4}$ in (2.18) and (2.19), we have

$$
\begin{gathered}
{\left[F_{3}(\widetilde{\rho}, \widetilde{u}, \widetilde{\ell}, \widetilde{\omega}, Q)\right](0, \cdot)=-m\left(\omega_{0} \times \ell_{0}\right)-\int_{\partial \mathcal{B}(0)} \sigma\left(u_{0}, p_{0}\right) n d \Gamma-k_{d} \ell_{0},} \\
{\left[F_{4}(\widetilde{\rho}, \widetilde{u}, \widetilde{\ell}, \widetilde{\omega}, Q)\right](0, \cdot)=-\int_{\partial \mathcal{B}(0)}\left(y-h_{0}\right) \times \sigma\left(u_{0}, p_{0}\right) n d \Gamma .}
\end{gathered}
$$

These expressions of $F_{3}(0, \cdot)$ and $F_{4}(0, \cdot)$ show that (1.16) gives the third condition $(2.32)$. We thus deduce from Proposition 2.2 the existence and uniqueness of $(\widehat{\rho}, \widehat{u}, \widehat{\ell}, \widehat{\omega}) \in \mathcal{S}_{T}$. Combining $(2.33)$, Proposition 2.4, (2.50) and (2.51), we obtain

$$
\|(\widehat{\rho}, \widehat{u}, \widehat{\ell}, \widehat{\omega})\|_{\mathcal{S}_{T}} \leqslant \frac{R}{2}+C T^{\alpha} .
$$

In particular, taking $T$ small enough, we deduce that $\mathcal{N}$ is well defined.

Next we show that $\mathcal{N}$ is a contraction. Let $\left(\widetilde{\rho}^{1}, \widetilde{u}^{1}, \widetilde{\ell}^{1}, \widetilde{\omega}^{1}\right),\left(\widetilde{\rho}^{2}, \widetilde{u}^{2}, \widetilde{\ell}^{2}, \widetilde{\omega}^{2}\right) \in \mathcal{S}_{T, R}$. For $j=1,2$, we set $\mathcal{N}\left(\widetilde{\rho}^{j}, \widetilde{u}^{j}, \widetilde{\ell}^{j}, \widetilde{\omega}^{j}\right):=\left(\widehat{\rho}^{j}, \widehat{u}^{j}, \widehat{\ell}^{j}, \widehat{\omega}^{j}\right)$. Using Proposition 2.2 and Proposition 2.4, we obtain

$$
\left\|\left(\widehat{\rho}^{1}, \widehat{u}^{1}, \widehat{\ell}^{1}, \widehat{\omega}^{1}\right)-\left(\widehat{\rho}^{2}, \widehat{u}^{2}, \widehat{\ell}^{2}, \widehat{\omega}^{2}\right)\right\|_{\mathcal{S}_{T}} \leqslant C T^{\alpha}\left\|\left(\widetilde{\rho}^{1}, \widetilde{u}^{1}, \widetilde{\ell}^{1}, \widetilde{\omega}^{1}\right)-\left(\widetilde{\rho}^{2}, \widetilde{u}^{2}, \widetilde{\ell}^{2}, \widetilde{\omega}^{2}\right)\right\|_{\mathcal{S}_{T}} .
$$

Thus $\mathcal{N}$ is a contraction in $\mathcal{S}_{T, R}$ for $T$ small enough.

Finally, using (2.51) and (2.39), we deduce

$$
\|(\widetilde{\rho}, \widetilde{u}, \widetilde{\ell}, \widetilde{\omega})\|_{\mathcal{S}_{T}}+\left\|h_{1}-h\right\|_{L^{\infty}(0, T)} \leqslant C \widetilde{\delta}_{0}=C\left(\left\|\rho_{0}-\bar{\rho}\right\|_{H^{3}}+\left\|u_{0}\right\|_{H^{3}}+\left|h_{1}-h_{0}\right|+\left|\ell_{0}\right|+\left|\omega_{0}\right|\right)
$$

that yields (2.3).

\section{Global in time existence of solutions}

3.1. A priori estimates. We have already established a local-in-time existence result in Theorem 2.1. In order to obtain the global in time existence of the solutions, we need an appropriate a priori estimates. We recall that $\|\cdot\|_{\widehat{\mathcal{S}}_{0, T}}$ is introduced in (1.13). We also introduce the following notation to shorten the notation: for $Z=L^{p}$ or $Z=W^{k, p}$, we set:

$$
\begin{gathered}
W_{T}^{k, \infty}(Z)=W^{k, \infty}(0, T ; Z(\mathcal{F}(t))), \quad H_{T}^{k}(Z)=H^{k}(0, T ; Z(\mathcal{F}(t))), \quad \text { for } \quad k=1,2, \\
W_{T}^{0, \infty}(Z)=L_{T}^{\infty}(Z)=L^{\infty}(0, T ; Z(\mathcal{F}(t))), \quad H_{T}^{0}(Z)=L_{T}^{2}(Z)=L^{2}(0, T ; Z(\mathcal{F}(t))) .
\end{gathered}
$$

The main tool to prove the global in time existence of the solutions is the following proposition:

Proposition 3.1. Let $h_{1} \in \Omega^{0}$ and $\bar{\rho}>0$. Assume the feedback law (1.10) with $\left(k_{p}, k_{d}\right)$ satisfying (1.17). There exist $\varepsilon_{0}, C_{0}>0$ with $\varepsilon_{0} \leqslant \delta_{0}$ such that if $(\rho, u, h, \ell, \omega)$ is a solution of system (1.1)-(1.10) with

$$
\|(\rho, u, \ell, \omega)\|_{\widehat{\mathcal{S}}_{0, T}} \leqslant \varepsilon_{0},
$$


then the following estimate holds:

$$
\|(\rho, u, \ell, \omega)\|_{\widehat{\mathcal{S}}_{0, T}}+\left\|\sqrt{k_{p}}\left(h_{1}-h\right)\right\|_{L^{\infty}(0, T)} \leqslant C_{0}\left(\left\|\left(\rho_{0}, u_{0}, \ell_{0}, \omega_{0}\right)\right\|_{\widehat{\mathcal{S}}_{0,0}}+\left|h_{1}-h_{0}\right|\right) .
$$

Proof. The proof follows closely the idea of [1, Proposition 8]. We only repeat some parts of the proof to estimate $\left(h_{1}-h\right)$. We define

$$
\rho^{*}(t, x)=\rho(t, x)-\bar{\rho}
$$

and we rewrite (1.1)-(1.9) as follows

$$
\begin{cases}\frac{\partial \rho^{*}}{\partial t}+u \cdot \nabla \rho^{*}+\bar{\rho} \operatorname{div} u=f_{0}\left(\rho^{*}, u, h, \omega\right) & t \in(0, T), x \in \mathcal{F}(t), \\ \frac{\partial u}{\partial t}-\operatorname{div} \sigma^{*}\left(u, \rho^{*}\right)=f_{1}\left(\rho^{*}, u, h, \omega\right) & t \in(0, T), x \in \mathcal{F}(t), \\ \bar{m} \ell^{\prime}=-\int_{\partial \mathcal{B}(t)} \sigma^{*}\left(u, \rho^{*}\right) N d \Gamma+\overline{k_{p}}\left(h_{1}-h(t)\right)-\overline{k_{d}} \ell(t)+f_{2}\left(\rho^{*}, u, h, \omega\right) & t \in(0, T), \\ \bar{J} \omega^{\prime}=-\int_{\partial \mathcal{B}(t)}(x-h) \times \sigma^{*}\left(u, \rho^{*}\right) N d \Gamma+f_{3}\left(\rho^{*}, u, h, \omega\right) & t \in(0, T), \\ h^{\prime}=\ell & t \in(0, T), \\ u(t, x)=0, & t \in(0, T), x \in \partial \Omega, \\ u(t, x)=\ell(t)+\omega(t) \times(x-h(t)), & t \in(0, T), x \in \partial \mathcal{B}(t), \\ \rho^{*}(0, \cdot)=\rho_{0}-\bar{\rho}, \quad u(0, \cdot)=u_{0} \quad \text { in } \quad \mathcal{F}(0), & \\ h(0)=h_{0}, \quad \ell(0)=\ell_{0}, \quad \omega(0)=\omega_{0}, & \end{cases}
$$

In the above system (3.3)

$$
\begin{gathered}
\bar{m}=\frac{m}{\bar{\rho}}, \quad \bar{J}=\frac{J}{\bar{\rho}}, \quad \overline{k_{p}}=\frac{k_{p}}{\bar{\rho}}, \quad \overline{k_{d}}=\frac{k_{d}}{\bar{\rho}}, \quad \bar{\mu}=\frac{\mu}{\bar{\rho}}, \quad \bar{\lambda}=\frac{\lambda}{\bar{\rho}}, \\
\sigma^{*}\left(u, \rho^{*}\right)=2 \bar{\mu} \mathbb{D}(u)+\bar{\lambda} \operatorname{div} u \mathbb{I}_{3}-p^{*} \rho^{*} \mathbb{I}_{3}, \quad p^{*}=a \gamma \bar{\rho}^{\gamma-2},
\end{gathered}
$$

and

$$
\left\{\begin{aligned}
f_{0}\left(\rho^{*}, u, h, \omega\right)= & -\rho^{*} \operatorname{div} u, \\
f_{1}\left(\rho^{*}, u, h, \omega\right)= & -(u \cdot \nabla) u-\left(\frac{1}{\bar{\rho}}-\frac{1}{\rho^{*}+\bar{\rho}}\right) \operatorname{div}\left(2 \mu \mathbb{D}(u)+\lambda \operatorname{div} u \mathbb{I}_{3}\right) \\
& +\left(p^{*}-a \gamma\left(\rho^{*}+\bar{\rho}\right)^{\gamma-2}\right) \nabla \rho^{*}, \\
f_{2}\left(\rho^{*}, u, h, \omega\right)= & -\int_{\partial \mathcal{B}(t)}\left(p^{*} \rho^{*}-\frac{a\left(\rho^{*}+\bar{\rho}\right)^{\gamma}}{\bar{\rho}}\right) N d \Gamma, \\
f_{3}\left(\rho^{*}, u, h, \omega\right)= & -\int_{\partial \mathcal{B}(t)}(x-h) \times\left(\left(p^{*} \rho^{*}-\frac{a\left(\rho^{*}+\bar{\rho}\right)^{\gamma}}{\bar{\rho}}\right) N\right) d \Gamma .
\end{aligned}\right.
$$

We take $\varepsilon_{0}$ small enough in (3.1) so that

$$
\rho^{*}+\bar{\rho} \geqslant \frac{\bar{\rho}}{2}
$$

After some calculations (that we skipped here), we obtain

$$
\begin{array}{r}
\left\|f_{0}\right\|_{L_{T}^{2}\left(H^{3}\right)}^{2}+\left\|f_{0}\right\|_{L_{T}^{\infty}\left(H^{2}\right)}^{2}+\left\|f_{0}\right\|_{H_{T}^{1}\left(L^{2}\right)}^{2}+\left\|f_{1}\right\|_{L_{T}^{2}\left(H^{2}\right)}^{2}+\left\|f_{1}\right\|_{L_{T}^{\infty}\left(H^{1}\right)}^{2}+\left\|f_{1}\right\|_{H_{T}^{1}\left(L^{2}\right)}^{2} \\
+\left\|f_{2}\right\|_{H^{1}(0, T)}^{2}+\left\|f_{3}\right\|_{H^{1}(0, T)}^{2} \leqslant C\|(\rho, u, \ell, \omega)\|_{\widehat{\mathcal{S}}_{0, T}}^{4},
\end{array}
$$


and

$$
\begin{aligned}
& \left\|\frac{\partial \rho^{*}}{\partial t}(0, \cdot)\right\|_{L^{2}}^{2}+\left\|\frac{\partial u}{\partial t}(0, \cdot)\right\|_{H^{1}}^{2}+\left|\ell^{\prime}(0)\right|^{2}+\left|\omega^{\prime}(0)\right|^{2} \\
& \leqslant C\left(\left\|\rho_{0}-\bar{\rho}\right\|_{H^{2}}^{2}+\left\|u_{0}\right\|_{H^{3}}^{2}+\left|\ell_{0}\right|^{2}+\left|h_{0}-h_{1}\right|^{2}+\left\|f_{0}\right\|_{L_{T}^{\infty}\left(L^{2}\right)}^{2}+\left\|f_{1}\right\|_{L_{T}^{\infty}\left(H^{1}\right)}^{2}+\left\|f_{2}\right\|_{L_{T}^{\infty}}^{2}+\left\|f_{3}\right\|_{L_{T}^{\infty}}^{2}\right) .
\end{aligned}
$$

In particular, if we can show

$$
\begin{array}{r}
\|(\rho, u, \ell, \omega)\|_{\widehat{\mathcal{S}}_{0, T}}^{2}+\left\|\sqrt{k_{p}}\left(h_{1}-h\right)\right\|_{L^{\infty}(0, T)}^{2} \leqslant C\left(\left\|f_{0}\right\|_{L_{T}^{2}\left(H^{3}\right)}^{2}+\left\|f_{0}\right\|_{L_{T}^{\infty}\left(H^{2}\right)}^{2}+\left\|f_{0}\right\|_{H_{T}^{1}\left(L^{2}\right)}^{2}+\left\|f_{1}\right\|_{L_{T}^{2}\left(H^{2}\right)}^{2}\right. \\
+\left\|f_{1}\right\|_{L_{T}^{\infty}\left(H^{1}\right)}^{2}+\left\|f_{1}\right\|_{H_{T}^{1}\left(L^{2}\right)}^{2}+\left\|f_{2}\right\|_{H^{1}(0, T)}^{2}+\left\|f_{3}\right\|_{H^{1}(0, T)}^{2}+\left\|\frac{\partial \rho^{*}}{\partial t}(0, \cdot)\right\|_{L^{2}}^{2}+\left\|\rho_{0}-\bar{\rho}\right\|_{H^{3}}^{2}+\left\|\frac{\partial u}{\partial t}(0, \cdot)\right\|_{H^{1}}^{2} \\
+\left\|u_{0}\right\|_{H^{3}(\mathcal{F}(0))}^{2}+\left|h_{0}-h_{1}\right|^{2}+\left|\ell^{\prime}(0)\right|^{2}+\left|\ell_{0}\right|^{2}+\left|\omega^{\prime}(0)\right|^{2}+\left|\omega_{0}\right|^{2}+\|(\rho, u, \ell, \omega)\|_{\widehat{\mathcal{S}}_{0, T}}^{3} \\
\left.+\|(\rho, u, \ell, \omega)\|_{\widehat{\mathcal{S}}_{0, T}}^{4}\right), \quad(3.4)
\end{array}
$$

then

$$
\begin{aligned}
\|(\rho, u, \ell, \omega)\|_{\widehat{\mathcal{S}}_{0, T}}^{2}+\left\|\sqrt{k_{p}}\left(h_{1}-h\right)\right\|_{L^{\infty}(0, T)}^{2} \leqslant & C\left(\|(\rho, u, \ell, \omega)\|_{\widehat{\mathcal{S}}_{0, T}}^{3}+\|(\rho, u, \ell, \omega)\|_{\widehat{\mathcal{S}}_{0, T}}^{4}\right. \\
& \left.+\left\|\rho_{0}-\bar{\rho}\right\|_{H^{3}}^{2}+\left\|u_{0}\right\|_{H^{3}}^{2}+\left|h_{0}-h_{1}\right|^{2}+\left|\ell_{0}\right|^{2}+\left|\omega_{0}\right|^{2}\right) .
\end{aligned}
$$

The condition (3.1) with $\varepsilon_{0}$ small enough combined with the above relation yields (3.2). The proof of (3.4) is done below.

The proof of (3.4) (that is necessary to finish the proof of Proposition 3.1) is done in a precise way in [1, Section 4] in the case $k_{p}=0$ and $k_{d}=0$. The presence of the corresponding terms only changes the two lemmas on time regularity (Lemma 13 and Lemma 14 in [1]). Here we state these two lemmas in our case and give the idea of their proofs with a particular attention to the feedback term. Then using these two lemmas and the elliptic results [1, Section 4], we can deduce (3.4) and thus end the proof of Proposition 3.1.

Lemma 3.2. Let $k=0,1$. For every $\varepsilon>0$, there exists a constant $C>0$ such that

$$
\begin{aligned}
& \left\|\rho^{*}\right\|_{W_{T}^{k, \infty}\left(L^{2}\right)}+\|u\|_{H_{T}^{k}\left(H^{1}\right)}+\|u\|_{W_{T}^{k, \infty}\left(L^{2}\right)}+\|\ell\|_{W^{k, \infty}(0, T)}+\|\ell\|_{H^{k}(0, T)}+\|\omega\|_{W^{k, \infty}(0, T)} \\
& +\left\|\sqrt{k_{p}}\left(h_{1}-h\right)\right\|_{L^{\infty}(0, T)} \leqslant \varepsilon\left(\left\|\rho^{*}\right\|_{H_{T}^{k}\left(L^{2}\right)}+\|\ell\|_{H^{k}(0, T)}+\|\omega\|_{H^{k}(0, T)}\right) \\
& +C\left(\left\|f_{0}\right\|_{H_{T}^{k}\left(L^{2}\right)}+\left\|f_{1}\right\|_{H_{T}^{k}\left(L^{2}\right)}+\left\|f_{2}\right\|_{H^{k}(0, T)}+\left\|f_{3}\right\|_{H^{k}(0, T)}\right. \\
& +\left\|\rho_{0}-\bar{\rho}\right\|_{L^{2}}+\left\|u_{0}\right\|_{L^{2}}+\left|h_{1}-h_{0}\right|+\left|\ell_{0}\right|+\left|\omega_{0}\right| \\
& \left.+\left\|\frac{\partial \rho^{*}}{\partial t}(0, \cdot)\right\|_{L^{2}}+\left\|\frac{\partial u}{\partial t}(0, \cdot)\right\|_{L^{2}}+\left|\ell^{\prime}(0)\right|+\left|\omega^{\prime}(0)\right|+\|(\rho, u, \ell, \omega)\|_{\widehat{\mathcal{S}}_{0, T}}^{3 / 2}+\|(\rho, u, \ell, \omega)\|_{\widehat{\mathcal{S}}_{0, T}}^{2}\right) .
\end{aligned}
$$


Proof of Lemma 3.2. Case $k=0$. We multiply equation $(3.3)_{1}$ by $p^{*} \rho^{*} / \bar{\rho},(3.3)_{2}$ by $u,(3.3)_{3}$ by $\ell$ and $(3.3)_{4}$ by $\omega$ :

$$
\begin{aligned}
& \int_{\mathcal{F}(t)}\left(\frac{p^{*}}{2 \bar{\rho}}\left|\rho^{*}\right|^{2}+\frac{|u|^{2}}{2}\right) d x+\int_{0}^{t} \int_{\mathcal{F}(s)}\left(2 \bar{\mu}|\mathbb{D}(u)|^{2}+\bar{\lambda}|\operatorname{div} u|^{2}\right) d x d s \\
&+ \frac{\bar{m}}{2}|\ell|^{2}+\frac{\bar{J}}{2}|\omega|^{2}+\frac{\bar{k}_{p}}{2}(t)\left|h_{1}-h(t)\right|^{2}+\bar{k}_{d} \int_{0}^{t}|\ell|^{2} d s \\
&= \int_{0}^{t} \int_{\mathcal{F}(s)}\left(f_{0} p^{*} \rho^{*} / \bar{\rho}+f_{1} \cdot u\right) d x d s+\int_{0}^{t}\left(f_{2} \cdot \ell+f_{3} \cdot \omega\right) d s \\
&+\int_{0}^{t} \int_{\mathcal{F}(s)}\left(\frac{p^{*}}{2 \bar{\rho}}\left|\rho^{*}\right|^{2} \operatorname{div} u+\operatorname{div}\left(\frac{|u|^{2} u}{2}\right)\right) d x d s \\
&+\int_{0}^{t} \frac{\bar{k}_{p}^{\prime}}{2}(s)\left|h_{1}-h(s)\right|^{2} d s+\int_{\mathcal{F}(0)} \frac{p^{*}}{2 \bar{\rho}}\left|\rho_{0}-\bar{\rho}\right|^{2} d y+\int_{\mathcal{F}(0)} \frac{\left|u_{0}\right|^{2}}{2} d y+\frac{\bar{m}}{2}\left|\ell_{0}\right|^{2}+\frac{\bar{J}}{2}\left|\omega_{0}\right|^{2} .
\end{aligned}
$$

Following standard calculation, we have

$$
\int_{0}^{t} \int_{\mathcal{F}(s)}\left(\frac{p^{*}}{2 \bar{\rho}}\left|\rho^{*}\right|^{2} \operatorname{div} u+\operatorname{div}\left(\frac{|u|^{2} u}{2}\right)\right) d x d s \leqslant C\|(\rho, u, \ell, \omega)\|_{\widehat{\mathcal{S}}_{0, T}}^{3} .
$$

It only remains to estimate

$$
\begin{aligned}
\int_{0}^{T} \frac{\bar{k}_{p}^{\prime}}{2}(s)\left|h_{1}-h(s)\right|^{2} d s \leqslant \int_{0}^{T_{I}} \frac{\bar{k}_{p}^{\prime}}{2}(s)\left|h_{1}-h(s)\right|^{2} d s & \\
& \leqslant\left\|\bar{k}_{p}^{\prime}\right\|_{L^{\infty}(0, T)}\left(T_{I}\left|h_{1}-h_{0}\right|^{2}+\int_{0}^{T_{I}}\left(\int_{0}^{s} \ell(z) d z\right)^{2} d s\right) .
\end{aligned}
$$

By using Hölder's inequality and (1.17), we obtain

$$
\int_{0}^{T} \frac{\bar{k}_{p}^{\prime}}{2}(s)\left|h_{1}-h(s)\right|^{2} d s \leqslant C\left|h_{1}-h_{0}\right|^{2}+\frac{k_{d}}{2} \int_{0}^{T}|\ell|^{2} d s .
$$

Combining (3.6), (3.7), (3.9) and Young's inequality, we deduce the result for $k=0$. 
Case $k=1$. By differentiating (3.3) with respect to $t$, we obtain:

$$
\begin{cases}\frac{\partial}{\partial t}\left(\frac{\partial \rho^{*}}{\partial t}\right)+(u \cdot \nabla) \frac{\partial \rho^{*}}{\partial t}+\bar{\rho} \operatorname{div} \frac{\partial u}{\partial t}=G_{0} & t \in(0, T), x \in \mathcal{F}(t), \\ \frac{\partial}{\partial t}\left(\frac{\partial u}{\partial t}\right)-\operatorname{div} \sigma^{*}\left(\frac{\partial u}{\partial t}, \frac{\partial \rho^{*}}{\partial t}\right)=G_{1} & t \in(0, T), x \in \mathcal{F}(t), \\ \bar{m} \ell^{\prime \prime}=-\int_{\partial \mathcal{B}(t)} \sigma^{*}\left(\frac{\partial u}{\partial t}, \frac{\partial \rho^{*}}{\partial t}\right) N d \Gamma+\left[\bar{k}_{p}\left(h_{1}-h(t)\right)\right]^{\prime}-\bar{k}_{d} \ell^{\prime}(t)+G_{2} & t \in(0, T), \\ \bar{J} \omega^{\prime \prime}=-\int_{\partial \mathcal{B}(t)}(x-h) \times \sigma^{*}\left(\frac{\partial u}{\partial t}, \frac{\partial \rho^{*}}{\partial t}\right) N d \Gamma+G_{3} & t \in(0, T), \\ h^{\prime}=\ell & t \in(0, T), \\ \frac{\partial u}{\partial t}(t, x)=0, & t \in(0, T), x \in \partial \Omega, \\ \frac{\partial u}{\partial t}(t, x)=\ell^{\prime}(t)+\omega^{\prime}(t) \times(x-h(t))+G_{4}, & t \in(0, T), x \in \partial \mathcal{B}(t) .\end{cases}
$$

where

$$
\left\{\begin{aligned}
G_{0} & =\frac{\partial f_{0}}{\partial t}-\left(\frac{\partial u}{\partial t} \cdot \nabla\right) \rho^{*}, \quad G_{1}=\frac{\partial f_{1}}{\partial t}, \quad G_{2}=\frac{\partial f_{2}}{\partial t}-\int_{\partial \mathcal{B}(t)} \ell \cdot \nabla\left(\sigma^{*}\left(u, \rho^{*}\right) N\right) d \Gamma, \\
G_{3} & \left.=\frac{\partial f_{3}}{\partial t}-\int_{\partial \mathcal{B}(t)} \ell \cdot \nabla\left((x-h) \times\left(\sigma^{*}\left(u, \rho^{*}\right)\right) N\right) d \Gamma+\int_{\partial \mathcal{B}(t)} \ell \times\left(\sigma^{*}\left(u, \rho^{*}\right)\right) N\right) d \Gamma \\
G_{4} & =-(\ell \cdot \nabla) u .
\end{aligned}\right.
$$

As in the first case, we multiply equation $(3.10)_{1}$ by $\frac{p *}{\bar{\rho}} \frac{\partial \rho^{*}}{\partial t}$, equation $(3.10)_{2}$ by $\frac{\partial u}{\partial t}$, equation (3.10) 3 by $\ell^{\prime}$, and equation $(3.10)_{4}$ by $\omega^{\prime}$. After some computations, we find

$$
\begin{aligned}
& \int_{\mathcal{F}(t)}\left(\frac{p^{*}}{2 \bar{\rho}}\left|\frac{\partial \rho^{*}}{\partial t}\right|^{2}+\frac{1}{2}\left|\frac{\partial u}{\partial t}\right|^{2}\right) d x+\int_{0}^{t} \int_{\mathcal{F}(s)}\left(2 \bar{\mu}\left|\mathbb{D}\left(\frac{\partial u}{\partial t}\right)\right|^{2}+\bar{\lambda}\left|\operatorname{div} \frac{\partial u}{\partial t}\right|^{2}\right) d x d s \\
& +\frac{\bar{m}}{2}\left|\ell^{\prime}(t)\right|^{2}+\frac{\bar{J}}{2}\left|\omega^{\prime}(t)\right|^{2}+\bar{k}_{d} \int_{0}^{t}\left|\ell^{\prime}(s)\right|^{2} d s \\
& =\int_{0}^{t} \int_{\mathcal{F}(s)}\left(G_{0} \frac{p^{*}}{\bar{\rho}} \frac{\partial \rho^{*}}{\partial t}+G_{1} \cdot \frac{\partial u}{\partial t}\right) d x d s+\int_{0}^{t}\left(G_{2} \cdot \ell^{\prime}+G_{3} \cdot \omega^{\prime}\right) d s+\int_{0}^{t} \int_{\partial \mathcal{B}(s)} G_{4} \cdot \sigma^{*}\left(\frac{\partial u}{\partial t}, \frac{\partial \rho^{*}}{\partial t}\right) N d \Gamma d s \\
& +\int_{0}^{t} \int_{\mathcal{F}(s)} \frac{p^{*}}{2 \bar{\rho}}\left|\frac{\partial \rho^{*}}{\partial t}\right|^{2} \operatorname{div} u d x d s+\int_{0}^{t} \int_{\partial \mathcal{F}(s)} \frac{1}{2} \operatorname{div}\left(\left|\frac{\partial u}{\partial t}\right|^{2} u\right) d x d s \\
& +\int_{0}^{t}\left[\bar{k}_{p}(s)\left(h_{1}-h(s)\right)\right]^{\prime} \cdot \ell^{\prime}(s) d s+\int_{\mathcal{F}(0)}\left(\frac{p^{*}}{2 \bar{\rho}}\left|\frac{\partial \rho^{*}}{\partial t}(0)\right|^{2}+\frac{1}{2}\left|\frac{\partial u}{\partial t}(0)\right|^{2}\right) d y+\frac{\bar{m}}{2}\left|\ell^{\prime}(0)\right|^{2}+\frac{\bar{J}}{2}\left|\omega^{\prime}(0)\right|^{2} .
\end{aligned}
$$


We have the following estimates as in [1, Lemma 13]:

$$
\begin{aligned}
&\left\|G_{0}\right\|_{L_{T}^{2}\left(L^{2}\right)}^{2}+\left\|G_{1}\right\|_{L_{T}^{2}\left(L^{2}\right)}^{2}+\left\|G_{2}\right\|_{L^{2}(0, T)}^{2}+\left\|G_{3}\right\|_{L^{2}(0, T)}^{2}+\left\|G_{4}\right\|_{L_{T}^{2}\left(L^{2}(\partial \mathcal{B}(t))\right.}^{2} \\
&+\int_{0}^{t} \int_{\mathcal{F}(s)}\left(\left|\frac{\partial \rho^{*}}{\partial t}\right|^{2} \operatorname{div} u+\operatorname{div}\left(\left|\frac{\partial u}{\partial t}\right|^{2} u\right)\right) d x d s \\
& \leqslant C\left(\left\|\frac{\partial f_{0}}{\partial t}\right\|_{L_{T}^{2}\left(L^{2}\right)}^{2}+\left\|\frac{\partial f_{1}}{\partial t}\right\|_{L_{T}^{2}\left(L^{2}\right)}^{2}+\left\|\frac{\partial f_{2}}{\partial t}\right\|_{L^{2}(0, T)}^{2}+\left\|\frac{\partial f_{3}}{\partial t}\right\|_{L^{2}(0, T)}^{2}\right. \\
&\left.+\|(\rho, u, \ell, \omega)\|_{\widehat{\mathcal{S}}_{0, T}}^{3}+\|(\rho, u, \ell, \omega)\|_{\widehat{\mathcal{S}}_{0, T}}^{4}\right)
\end{aligned}
$$

It only remains to estimate the term coming from the feedback:

$$
\begin{aligned}
\int_{0}^{t}\left[\bar{k}_{p}(s)\left(h_{1}-h(s)\right)\right]^{\prime} \cdot \ell^{\prime}(s) d s= & \int_{0}^{t} \bar{k}_{p}^{\prime}(s)\left(h_{1}-h(s)\right) \cdot \ell^{\prime}(s) d s-\int_{0}^{t} \bar{k}_{p}(s) \ell(s) \cdot \ell^{\prime}(s) d s \\
& =\int_{0}^{t} \bar{k}_{p}^{\prime}(s)\left(h_{1}-h(s)\right) \cdot \ell^{\prime}(s) d s+\int_{0}^{t} \frac{\bar{k}_{p}^{\prime}}{2}(s)|\ell(s)|^{2} d s-\frac{\bar{k}_{p}}{2}(t)|\ell(t)|^{2},
\end{aligned}
$$

and proceeding as in (3.8), we have the following estimates

$$
\int_{0}^{t}\left[\bar{k}_{p}(s)\left(h_{1}-h(s)\right)\right]^{\prime} \cdot \ell^{\prime}(s) d s \leqslant C\left(\left|h_{1}-h_{0}\right|^{2}+\int_{0}^{T}|\ell(s)|^{2} d s\right)+\frac{\bar{k}_{d}}{2} \int_{0}^{T}\left|\ell^{\prime}(s)\right|^{2} d s .
$$

We can estimate $\|\ell\|_{L^{2}(0, T)}^{2}$ with (3.5) for $k=0$. With this remark and combining (3.11), inequality (3.13) and the above estimates we deduce (3.5) for $k=1$.

Lemma 3.3. Let $k=0,1$. There exists a constant $C>0$ such that

$$
\begin{array}{r}
\left\|\frac{\partial \rho^{*}}{\partial t}\right\|_{H_{T}^{k}\left(L^{2}\right)}+\|u\|_{W_{T}^{k, \infty}\left(H^{1}\right)}+\left\|\frac{\partial u}{\partial t}\right\|_{H_{T}^{k}\left(L^{2}\right)}+\left\|\ell^{\prime}\right\|_{H^{k}(0, T)}+\left\|\omega^{\prime}\right\|_{H^{k}(0, T)} \\
\leqslant C\left(\left\|\rho^{*}\right\|_{W_{T}^{k, \infty}\left(L^{2}\right)}+\|u\|_{H_{T}^{k}\left(H^{1}\right)}+\|\ell\|_{W^{k}, \infty(0, T)}+\|\ell\|_{H^{k}(0, T)}+\left\|\sqrt{k_{p}}\left(h_{1}-h\right)\right\|_{L^{\infty}(0, T)}\right. \\
+\left\|f_{0}\right\|_{H_{T}^{k}\left(L^{2}\right)}+\left\|f_{1}\right\|_{H_{T}^{k}\left(L^{2}\right)}+\left\|f_{2}\right\|_{H^{k}(0, T)}+\left\|f_{3}\right\|_{H^{k}(0, T)} \\
+\left\|\rho_{0}-\bar{\rho}\right\|_{L^{2}}+\left\|\frac{\partial \rho}{\partial t}(0, \cdot)\right\|_{L^{2}}+\left\|u_{0}\right\|_{H^{1}}+\left\|\frac{\partial u}{\partial t}(0, \cdot)\right\|_{H^{1}}+\left|h_{1}-h_{0}\right| \\
\left.+\left|\ell_{0}\right|+\left|\ell^{\prime}(0)\right|+\left|\omega_{0}\right|+\|(\rho, u, \ell, \omega)\|_{\widehat{\mathcal{S}}_{0, T}}^{3 / 2}+\|(\rho, u, \ell, \omega)\|_{\widehat{\mathcal{S}}_{0, T}}^{2}\right) .
\end{array}
$$


Proof of Lemma 3.3. Case $k=0$. We multiply equation $(3.3)_{1}$ by $\frac{\partial \rho^{*}}{\partial t},(3.3)_{2}$ by $\frac{\partial u}{\partial t},(3.3)_{3}$ by $\ell^{\prime}$ and $(3.3)_{4}$ by $\omega^{\prime}$. After standard computations, we find

$$
\begin{gathered}
\int_{0}^{t} \int_{\mathcal{F}(s)}\left(\left|\frac{\partial \rho^{*}}{\partial t}\right|^{2}+\left|\frac{\partial u}{\partial t}\right|^{2}\right) d x d s \\
+\int_{\mathcal{F}(t)}\left(2 \bar{\mu}|\mathbb{D}(u)|^{2}+\bar{\lambda}|\operatorname{div} u|^{2}\right) d x+\int_{0}^{t}\left(\bar{m}\left|\ell^{\prime}\right|^{2}+\bar{J}\left|\omega^{\prime}\right|^{2}\right) d s \\
\quad+\bar{k}_{d}|\ell(t)|^{2}-2 \int_{0}^{t} \bar{k}_{p}\left(h_{1}-h\right) \cdot \ell^{\prime} d s \\
\leqslant C\left(\int_{0}^{t} \int_{\mathcal{F}(s)}\left|\operatorname{div}\left(\left(2 \bar{\mu}|\mathbb{D}(u)|^{2}+\bar{\lambda}|\operatorname{div} u|^{2}\right) u\right)\right| d x d s+\int_{0}^{t} \int_{\mathcal{F}(s)}\left(p^{*} \rho^{*} \operatorname{div} \frac{\partial u}{\partial t}-\bar{\rho} \frac{\partial \rho^{*}}{\partial t} \operatorname{div} u\right) d x d s\right. \\
+\int_{0}^{t} \int_{\mathcal{F}(s)}\left|\frac{\partial \rho^{*}}{\partial t}\right|\left|\left(u \cdot \nabla \rho^{*}\right)\right| d x d s+\int_{\mathcal{F}(0)}\left|\nabla u_{0}\right|^{2} d x+\bar{k}_{d}\left|\ell_{0}\right|^{2} \\
\left.+\int_{0}^{t}\left(\int_{\mathcal{F}(s)}\left(\left|f_{0}\right|^{2}+\left|f_{1}\right|^{2}\right) d x+\left|f_{2}\right|^{2}+\left|f_{3}\right|^{2}\right) d s+\int_{0}^{t} \int_{\partial \mathcal{B}(s)} G_{4} \cdot \sigma^{*}\left(u, \rho^{*}\right) N d \Gamma d s\right)
\end{gathered}
$$

The terms in the right-hand side of (3.15) can be estimated as in Lemma 14 in [1]. We only estimate

$$
-\int_{0}^{t} \bar{k}_{p}\left(h_{1}-h\right) \cdot \ell^{\prime} d s=-\bar{k}_{p}(t)\left(h_{1}-h\right) \cdot \ell(t)+\int_{0}^{t}\left(\bar{k}_{p}^{\prime}\left(h_{1}-h\right) \cdot \ell-\bar{k}_{p}|\ell|^{2}\right) d s
$$

and thus

$$
\left|-\int_{0}^{t} \bar{k}_{p}\left(h_{1}-h\right) \cdot \ell^{\prime} d s\right| \leqslant C\left(\left|h_{1}-h_{0}\right|^{2}+k_{p}\left|h_{1}-h\right|^{2}+|\ell|^{2}+\int_{0}^{t}|\ell(s)|^{2} d s\right) .
$$


$\underline{\text { Case } k=1}$. We multiply $(3.10)_{1}$ by $\frac{\partial^{2} \rho^{*}}{\partial t^{2}},(3.10)_{2}$ by $\frac{\partial^{2} u}{\partial t^{2}}(3.10)_{3}$ by $\ell^{\prime \prime}$ and $(3.10)_{4}$ by $\omega^{\prime \prime}$. Following the proof of Lemma 14 in [1], we find

$$
\begin{aligned}
& \int_{0}^{t} \int_{\mathcal{F}(s)}\left(\left|\frac{\partial^{2} \rho^{*}}{\partial t^{2}}\right|^{2}+\left|\frac{\partial^{2} u}{\partial t^{2}}\right|^{2}\right) d x d s+\int_{\mathcal{F}(t)}\left(2 \bar{\mu}\left|\mathbb{D}\left(\frac{\partial u}{\partial t}\right)\right|^{2}+\bar{\lambda}\left|\operatorname{div} \frac{\partial u}{\partial t}\right|^{2}\right) d x \\
&+\int_{0}^{t}\left(\frac{\bar{m}}{2}\left|\ell^{\prime \prime}\right|^{2}+\frac{\bar{J}}{2}\left|\omega^{\prime \prime}\right|^{2}\right) d s+2 \bar{k}_{d}\left(\left|\ell^{\prime}(t)\right|^{2}-\left|\ell^{\prime}(0)\right|^{2}\right) \\
& \quad-\int_{0}^{t} \bar{k}_{p}^{\prime}(s)\left(h_{1}-h(s)\right) \cdot \ell^{\prime \prime}(s) d s+\int_{0}^{t} \bar{k}_{p}(s) \ell(s) \cdot \ell^{\prime \prime}(s) d s \\
& \leqslant C\left(\left\|\rho^{*}\right\|_{W_{T}^{1, \infty}\left(L^{2}\right)}^{2}+\|u\|_{H_{T}^{1}\left(H^{1}\right)}^{2}+\|\ell\|_{W^{1, \infty}(0, T)}^{2}+\|\ell\|_{H^{1}(0, T)}^{2}\right. \\
&+\left\|f_{0}\right\|_{H_{T}^{1}\left(L^{2}\right)}^{2}+\left\|f_{1}\right\|_{H_{T}^{1}\left(L^{2}\right)}^{2}+\left\|f_{2}\right\|_{H^{1}(0, T)}^{2}+\left\|f_{3}\right\|_{H^{1}(0, T)}^{2} \\
&+\left\|\rho_{0}-\bar{\rho}\right\|_{L^{2}}^{2}+\left\|\frac{\partial \rho}{\partial t}(0, \cdot)\right\|_{L^{2}}^{2}+\left\|u_{0}\right\|_{H^{1}}^{2}+\left\|\frac{\partial u}{\partial t}(0, \cdot)\right\|_{H^{1}}^{2} \\
&\left.+\left|\ell_{0}\right|^{2}+\left|\ell^{\prime}(0)\right|^{2}+\left|\omega_{0}\right|^{2}+\|(\rho, u, \ell, \omega)\|_{\widehat{\mathcal{S}}_{0, T}}^{3}+\|(\rho, u, \ell, \omega)\|_{\widehat{\mathcal{S}}_{0, T}}^{4}\right) .
\end{aligned}
$$

We estimate the additional term due to the feedback:

$$
\begin{aligned}
& \left|\int_{0}^{t}\left(-\bar{k}_{p}^{\prime}(s)\left(h_{1}-h(s)\right) \cdot \ell^{\prime \prime}(s)+\bar{k}_{p}(s) \ell(s) \cdot \ell^{\prime \prime}(s)\right) d s\right| \\
& \leqslant C\left(\left|h_{1}-h_{0}\right|^{2}+\int_{0}^{T}|\ell(s)|^{2} d s\right)+\frac{\bar{m}}{4} \int_{0}^{t}\left|\ell^{\prime \prime}(s)\right|^{2} d s,
\end{aligned}
$$

and this allows us to prove this case.

\subsection{Proof of Theorem 1.1.}

Proof. We combine Theorem 2.1 and Proposition 3.1 to establish our result. Note that we can take $\delta_{0}$ small enough in Theorem 2.1 so that $(2.2)$ yields

$$
h_{0} \in \Omega^{0} \text { and } \rho_{0}>0 .
$$

Since $h_{1} \in \Omega^{0}$, there exists $\eta>0$ such that

$$
\operatorname{dist}\left(h_{1}, \partial \Omega\right)>1+2 \eta .
$$

We can assume that $\delta_{0} \leqslant \eta$ where $\delta_{0}$ is the constant in (2.2).

Let us fix

$$
\delta=\min \left(\delta_{0}, \frac{\varepsilon_{0}}{C_{*}}, \frac{\varepsilon_{0}}{C_{0}\left(1+\frac{C_{*}}{\sqrt{k_{p}\left(T_{*}\right)}}\right)}, \frac{\eta \sqrt{k_{p}\left(T_{*}\right)}}{C_{0}}\right),
$$


where the constants $\delta_{0}, C_{*}$ are appeared in Theorem 2.1, $\varepsilon_{0}, C_{0}$ are introduced in Proposition 3.1. Since $\left(\rho_{0}, u_{0}, h_{0}, \ell_{0}, \omega_{0}\right)$ satisfies (1.18)-(1.19) and $\delta \leqslant \delta_{0}$, we can apply Theorem 2.1 to obtain the existence of solution of system (1.1)-(1.10) in $\left(0, T_{*}\right)$ and

$$
\|(\rho, u, \ell, \omega)\|_{\widehat{\mathcal{S}}_{0, T_{*}}}+\left\|h_{1}-h\right\|_{L^{\infty}\left(0, T_{*}\right)} \leqslant C_{*}\left(\left\|\left(\rho_{0}, u_{0}, \ell_{0}, \omega_{0}\right)\right\|_{\widehat{\mathcal{S}}_{0,0}}+\left|h_{1}-h_{0}\right|\right) .
$$

In particular, from (1.19) and (3.19),

$$
\|(\rho, u, \ell, \omega)\|_{\widehat{\mathcal{S}}_{0, T_{*}}}+\left\|h_{1}-h\right\|_{L^{\infty}\left(0, T_{*}\right)} \leqslant C_{*} \delta \leqslant \varepsilon_{0} \leqslant \delta_{0} .
$$

Thus $\operatorname{dist}(h(t), \partial \Omega)>1+\eta$ for $t \in\left[0, T_{*}\right]$ and Proposition 3.1 gives

$$
\|(\rho, u, \ell, \omega)\|_{\widehat{\mathcal{S}}_{0, T_{*}}}+\left\|\sqrt{k_{p}}\left(h_{1}-h\right)\right\|_{L^{\infty}\left(0, T_{*}\right)} \leqslant C_{0}\left(\left\|\left(\rho_{0}, u_{0}, \ell_{0}, \omega_{0}\right)\right\|_{\widehat{\mathcal{S}}_{0,0}}+\left|h_{1}-h_{0}\right|\right) .
$$

Using that $(\rho, u, h, \ell, \omega)$ is solution of (1.1)-(1.10), one can check that

$$
\left(\rho\left(T_{*}, \cdot\right), u\left(T_{*}, \cdot\right), h\left(T_{*}\right), \ell\left(T_{*}\right), \omega\left(T_{*}\right)\right)
$$

satisfies the compatibility conditions (1.14)-(1.16) and, from (3.20), we have

$$
\left\|\left(\rho\left(T_{*}, \cdot\right), u\left(T_{*}, \cdot\right), \ell\left(T_{*}\right), \omega\left(T_{*}\right)\right)\right\|_{\widehat{\mathcal{S}}_{T_{*}, T_{*}}}+\left|h_{1}-h\left(T_{*}\right)\right| \leqslant \delta_{0} .
$$

We can thus apply again Theorem 2.1 to extend our solution on $\left(T_{*}, 2 T_{*}\right)$ and using (3.21), we find

$$
\begin{aligned}
\|(\rho, u, \ell, \omega)\|_{\widehat{\mathcal{S}}_{T_{*}, 2 T_{*}}} & +\left\|\sqrt{k_{p}}\left(h_{1}-h\right)\right\|_{L^{\infty}\left(T_{*}, 2 T_{*}\right)} \\
& \leqslant C_{*}\left(\left\|\left(\rho\left(T_{*}, \cdot\right), u\left(T_{*}, \cdot\right), \ell\left(T_{*}\right), \omega\left(T_{*}\right)\right)\right\|_{\widehat{\mathcal{S}}_{T_{*}, T_{*}}}+\left|h_{1}-h\left(T_{*}\right)\right|\right) \\
& \leqslant \frac{C_{*} C_{0}}{\sqrt{k_{p}\left(T_{*}\right)}}\left(\left\|\left(\rho_{0}, u_{0}, \ell_{0}, \omega_{0}\right)\right\|_{\widehat{\mathcal{S}}_{0,0}}+\left|h_{1}-h_{0}\right|\right) .
\end{aligned}
$$

Thus, combining (3.21) and (3.22), and using (3.19), we obtain

$$
\begin{array}{r}
\|(\rho, u, \ell, \omega)\|_{\widehat{\mathcal{S}}_{0,2 T *}}+\left\|\sqrt{k_{p}}\left(h_{1}-h\right)\right\|_{L^{\infty}\left(0,2 T_{*}\right)} \leqslant C_{0}\left(1+\frac{C_{*}}{\sqrt{k_{p}\left(T_{*}\right)}}\right)\left(\left\|\left(\rho_{0}, u_{0}, \ell_{0}, \omega_{0}\right)\right\|_{\widehat{\mathcal{S}}_{0,0}}+\left|h_{1}-h_{0}\right|\right) \\
\leqslant C_{0}\left(1+\frac{C_{*}}{\sqrt{k_{p}\left(T_{*}\right)}}\right) \delta \leqslant \varepsilon_{0} .
\end{array}
$$

Applying Proposition 3.1, we deduce

$$
\|(\rho, u, \ell, \omega)\|_{\widehat{\mathcal{S}}_{0,2 T_{*}}}+\left\|\sqrt{k_{p}}\left(h_{1}-h\right)\right\|_{L^{\infty}\left(0,2 T_{*}\right)} \leqslant C_{0}\left(\left\|\left(\rho_{0}, u_{0}, \ell_{0}, \omega_{0}\right)\right\|_{\widehat{\mathcal{S}}_{0,0}}+\left|h_{1}-h_{0}\right|\right) .
$$

In particular $\operatorname{dist}(h(t), \partial \Omega)>1+\eta$ for $t \in\left[T_{*}, 2 T_{*}\right]$. Moreover, from (3.22) and (3.19),

$$
\left\|\left(\rho\left(2 T_{*}, \cdot\right), u\left(2 T_{*}, \cdot\right), \ell\left(2 T_{*}\right), \omega\left(2 T_{*}\right)\right)\right\|_{\widehat{\mathcal{S}}_{2 T_{*}, 2 T_{*}}}+\left|h_{1}-h\left(2 T_{*}\right)\right| \leqslant C_{0} \frac{C_{*}}{\sqrt{k_{p}\left(T_{*}\right)}} \delta \leqslant \varepsilon_{0} \leqslant \delta_{0} .
$$

Then, we repeat the argument on $\left[j T_{*},(j+1) T_{*}\right], j \in \mathbb{N}^{*}$ and we use that $k_{p}$ is non-decreasing to conclude the proof. 


\section{Proof of Theorem 1.2}

This section is devoted to the proof of Theorem 1.2. First, from Theorem 1.1, we have

$$
\rho-\bar{\rho} \in H^{1}\left(0, \infty ; H^{2}(\mathcal{F}(t))\right), \quad u \in H^{1}\left(0, \infty ; H^{2}(\mathcal{F}(t)), \quad \ell, \omega \in H^{2}(0, \infty)\right.
$$

so that ([3, Corollary 8.9, p.214]),

$$
\lim _{t \rightarrow \infty}\|\rho(t, .)-\bar{\rho}\|_{H^{2}(\mathcal{F}(t))}=0, \quad \lim _{t \rightarrow \infty}\|u(t, .)\|_{H^{2}(\mathcal{F}(t))}=0, \quad \lim _{t \rightarrow \infty} \ell(t)=0, \quad \lim _{t \rightarrow \infty} \omega(t)=0 .
$$

In the rest of the section, we show $\lim _{t \rightarrow \infty} h(t)=h_{1}$ that completes the proof of Theorem 1.2. In order to do this, we need the notion of weak solutions for the problem (1.1)-(1.9). First, we extend $\rho$ and $u$ in $\mathbb{R}^{3}$ by the formula

$$
\rho=\left\{\begin{array}{l}
\rho \text { in } \mathcal{F}(t), \\
\frac{3 m}{4 \pi}=\rho_{\mathcal{B}} \text { in } \mathcal{B}(t), \\
0 \text { in } \mathbb{R}^{3} \backslash \Omega .
\end{array} \quad u=\left\{\begin{array}{l}
u \text { in } \mathcal{F}(t), \\
\ell(t)+\omega(t) \times(x-h(t))=u_{\mathcal{B}} \text { in } \mathcal{B}(t), \\
0 \text { in } \mathbb{R}^{3} \backslash \Omega .
\end{array}\right.\right.
$$

Then we consider the following notion of weak solutions (see [9]).

Definition 4.1. A triplet $(\rho, u, h)$ is a weak solution to (1.1)-(1.9) on $(0, T)$ if

$$
\begin{gathered}
\rho \geqslant 0, \quad \rho \in L^{\infty}\left(0, T ; L^{\gamma}(\Omega)\right) \cap C\left([0, T] ; L^{1}(\Omega)\right), \quad u \in L^{2}\left(0, T ; H_{0}^{1}(\Omega)\right), \\
u=\ell(t)+\omega(t) \times(x-h(t)) \text { in } \mathcal{B}(t), \quad h^{\prime}=\ell, \\
\int_{0}^{T} \int_{\mathbb{R}^{3}}\left[\rho \frac{\partial \phi}{\partial t}+(\rho u) \cdot \nabla \phi\right] d x d t=0, \\
\int_{0}^{T} \int_{\mathbb{R}^{3}}\left[b(\rho) \frac{\partial \phi}{\partial t}+(b(\rho) u) \cdot \nabla \phi+\left(b(\rho)-b^{\prime}(\rho) \rho\right) \operatorname{div} u \phi\right] d x d t=0,
\end{gathered}
$$

for any $\phi \in C_{c}^{\infty}\left((0, T) \times \mathbb{R}^{3}\right)$ and for any $b \in C^{1}(\mathbb{R})$ such that $b^{\prime}(z)=0$ for $z$ large enough;

$$
\begin{aligned}
\int_{0}^{T} \int_{\mathbb{R}^{3}}\left[(\rho u) \cdot \frac{\partial \phi}{\partial t}+(\rho u \otimes u): \mathbb{D}(\phi)+a \rho^{\gamma} \operatorname{div} \phi\right] d x d t \\
\quad=\int_{0}^{T} \int_{\mathbb{R}^{3}}\left(2 \mu \mathbb{D}(u)+\lambda \operatorname{div} u \mathbb{I}_{3}\right): \mathbb{D}(\phi) d x d t+\int_{0}^{T} w \cdot \ell_{\phi} d t,
\end{aligned}
$$

for any $\phi \in C_{c}^{\infty}((0, T) \times \Omega)$, with $\phi(t, y)=\ell_{\phi}(t)+\omega_{\phi}(t) \times(y-h(t))$ in a neighborhood of $\mathcal{B}(t)$; for a.e. $t \in[0, T]$, the following energy inequality holds:

$$
\begin{aligned}
\int_{\Omega}\left(\frac{\rho(t, x)}{2}|u(t, x)|^{2}+\frac{a}{\gamma-1} \rho^{\gamma}(t, x)\right) d x & +\int_{0}^{t} \int_{\Omega}\left(2 \mu|D(u)|^{2}+\lambda|\operatorname{div} u|^{2}\right) d x d t \\
& \leqslant C\left(\int_{\{\rho(0)>0\}}\left(\frac{1}{2} \frac{|q(x)|^{2}}{\rho(0, x)}+\frac{a}{\gamma-1} \rho^{\gamma}(0, x)\right) d x+\int_{0}^{t} w \cdot \ell d t\right) ;
\end{aligned}
$$


and

$$
\rho(0, \cdot)=\rho_{0}, \quad(\rho u)(0, \cdot)=q, \quad h(0)=h_{0} .
$$

We now state a result on the weak compactness of the set of weak solutions to the problem (1.1)-(1.9) obtained in [9, Theorem 9.1].

Theorem 4.2. Let $\left(\rho_{n}, u_{n}, h_{n}\right)$ be a sequence of weak solutions to (1.1)-(1.9) on $(0, T) \times \Omega$ with the initial condition $\left(\rho_{0, n}, u_{0, n}, h_{0, n}\right)$ and forcing term $w_{n}$ for each $n \geqslant 1$. Assume that $\left\{w_{n}\right\}$ is a sequence of bounded and measurable functions such that

$$
w_{n} \rightarrow w \text { weakly * in } L^{\infty}(0, T)
$$

along with

$$
\begin{array}{rcc}
\rho_{0 n} \rightarrow \rho_{0} \quad \text { in } & L^{\gamma}\left(\mathbb{R}^{3}\right), \\
\rho_{0 n} u_{0 n}=q_{n} \rightarrow q \quad \text { in } & L^{1}\left(\mathbb{R}^{3}\right),
\end{array}
$$

where $\rho_{0}, q$ satisfy the following compatibility conditions

$$
q=0 \text { a.e. on the set }\left\{x \in \Omega \mid \rho_{0}=0\right\}, \frac{|q|^{2}}{\rho_{0}} \in L^{1}(\Omega) .
$$

Moreover, let

$$
\int_{\left\{\rho_{0 n}>0\right\}}\left(\frac{1}{2} \frac{\left|q_{n}\right|^{2}}{\rho_{0 n}}+\frac{a}{\gamma-1} \rho_{n}^{\gamma}(0)\right) d x \rightarrow \int_{\left\{\rho_{0}>0\right\}}\left(\frac{1}{2} \frac{|q|^{2}}{\rho_{0}}+\frac{a}{\gamma-1} \rho_{0}^{\gamma}\right) d x
$$

and

$$
h_{0 n} \rightarrow h_{0}
$$

Then there is a subsequence such that

$$
\begin{array}{r}
\rho_{n} \rightarrow \rho \text { in } C\left([0, T] ; L^{1}\left(\mathbb{R}^{3}\right)\right), \\
u_{n} \rightarrow u \quad \text { weakly in } L^{2}\left(0, T ; H_{0}^{1}(\Omega)\right), \\
h_{n} \rightarrow h \text { uniformly in }(0, T) .
\end{array}
$$

where $(\rho, u, h)$ is a weak solution of the problem $(1.1)-(1.9)$ on $(0, T) \times \Omega$ with the initial conditions $\left(\rho_{0}, q, h_{0}\right)$.

With the help of above result, we can now prove Theorem 1.2.

Proof of Theorem 1.2. From (1.21), there exist $h^{*} \in \Omega^{0}$ and $\left\{t_{n}\right\} \subset \mathbb{R}_{+}^{*}$ such that

$$
t_{n} \rightarrow \infty, \quad \lim _{n \rightarrow \infty} h\left(t_{n}\right)=h^{*} .
$$

Define

Writing

$$
\rho^{*}=\mathbb{1}_{\widehat{\mathcal{F}}\left(h^{*}\right)} \bar{\rho}+\mathbb{1}_{\widehat{\mathcal{B}}\left(h^{*}\right)} \rho_{\mathcal{B}} .
$$

$$
\rho\left(t_{n}, \cdot\right)-\rho^{*}=\left[\rho\left(t_{n}\right)-\bar{\rho}\right] \mathbb{1}_{\mathcal{F}\left(t_{n}\right)}+\bar{\rho}\left[\mathbb{1}_{\mathcal{F}\left(t_{n}\right)}-\mathbb{1}_{\widehat{\mathcal{F}}\left(h^{*}\right)}\right]+\rho_{\mathcal{B}}\left[\mathbb{1}_{\mathcal{B}\left(t_{n}\right)}-\mathbb{1}_{\widehat{\mathcal{B}}\left(h^{*}\right)}\right],
$$

and using (4.1), we deduce

$$
\begin{aligned}
& \rho\left(t_{n}, \cdot\right) \stackrel{t_{n} \rightarrow \infty}{\longrightarrow} \rho^{*} \quad \text { in } \quad L^{\gamma}\left(\mathbb{R}^{3}\right), \\
& \rho\left(t_{n}, \cdot\right) u\left(t_{n}, \cdot\right) \stackrel{t_{n} \rightarrow \infty}{\longrightarrow} 0 \quad \text { in } \quad L^{1}\left(\mathbb{R}^{3}\right), \\
& \rho\left(t_{n}, \cdot\right)\left|u\left(t_{n}, \cdot\right)\right|^{2} \stackrel{t_{n} \rightarrow \infty}{\longrightarrow} 0 \quad \text { in } \quad L^{1}\left(\mathbb{R}^{3}\right) \text {. }
\end{aligned}
$$


We set

$$
\rho_{0 n}=\rho\left(t_{n}\right), \quad u_{0 n}=u\left(t_{n}\right), \quad h_{0 n}=h\left(t_{n}\right),
$$

that satisfy (4.3), (4.4), (4.5), (4.6) and (4.7) with $\left\{\rho_{0 n}>0\right\}=\left\{\rho_{0}>0\right\}=\Omega$. We also define

$$
\rho_{n}(t, x)=\rho\left(t+t_{n}, x\right), \quad u_{n}(t, x)=u\left(t+t_{n}, x\right), \quad h_{n}(t)=h\left(t+t_{n}\right), \quad \ell_{n}(t)=\ell\left(t+t_{n}\right),
$$

that is a weak solution to (1.1)-(1.9) in the sense of Definition 4.1 (since it is a strong solution) with initial conditions $\left(\rho_{0 n}, u_{0 n}, h_{0 n}\right)$ and with

$$
w_{n}(t)=k_{p}(t)\left(h_{1}-h_{n}(t)\right)-k_{d} \ell_{n}(t) .
$$

From Theorem 1.1, we have that

$$
w_{n} \rightarrow \widehat{w} \text { weakly } * \text { in } L^{\infty}(0, T) .
$$

Thus, we can apply Theorem 4.2 and we deduce that up to a subsequence for $T>0$ :

$$
\begin{aligned}
& \rho_{n} \rightarrow \widehat{\rho} \quad \text { in } \quad C\left([0, T] ; L^{1}\left(\mathbb{R}^{3}\right)\right), \\
& u_{n} \rightarrow \widehat{u} \quad \text { weakly in } L^{2}\left(0, T ; H_{0}^{1}(\Omega)\right), \\
& h_{n} \rightarrow \widehat{h} \quad \text { in } \quad L^{\infty}(0, T),
\end{aligned}
$$

with $(\widehat{\rho}, \widehat{u}, \widehat{h})$ is a weak solution of (1.1)-(1.9) such that

$$
\widehat{\rho}(0, \cdot)=\rho^{*}, \quad(\widehat{\rho} \widehat{u})(0, \cdot)=0, \quad \widehat{h}(0)=h^{*},
$$

and with

$$
\widehat{w}(t)=k_{p}(t)\left(h_{1}-\widehat{h}(t)\right)-k_{d} \widehat{\ell}(t)
$$

Moreover up to a subsequence,

$$
\int_{0}^{T}\left\|\mathbb{D}\left(u_{n}(t, \cdot)\right)\right\|_{L^{2}(\Omega)}^{2} d t=\int_{t_{n}}^{t_{n}+T}\|\mathbb{D}(u(t, \cdot))\|_{L^{2}(\Omega)}^{2} d t \stackrel{n \rightarrow \infty}{\longrightarrow} 0 .
$$

The above limit and (4.8) yield

$$
\mathbb{D} \widehat{u}=0 \text { in }(0, T) \times \Omega .
$$

Thus, we deduce that $\widehat{u}=0$ in $(0, T) \times \Omega$. In particular, we have $\widehat{h}^{\prime}(t)=0, \forall t \in(0, T)$. This gives,

$$
\widehat{h}=h^{*} \text { in }(0, T) \text {. }
$$

Consequently, (4.2) gives

$$
\int_{0}^{T} \int_{\mathbb{R}^{3}} a(\widehat{\rho})^{\gamma} \operatorname{div} \phi d x d t=\int_{0}^{T} k_{p}\left(h_{1}-h^{*}\right) \cdot \ell_{\phi} d t,
$$

for all $\phi \in C_{c}^{\infty}((0, T) \times \Omega)$, with $\phi(t, y)=\ell_{\phi}(t)+\omega_{\phi}(t) \times(y-h(t))$ in a neighborhood of $\mathcal{B}(t)$. Then we take

$$
\operatorname{div} \phi=0, \phi(t, \cdot)=\left(h_{1}-h^{*}\right) \zeta(t) \text { in } \mathcal{B}(t), \text { with } \zeta \in C_{c}^{\infty}((0, T)),
$$

so that

$$
\int_{0}^{T}\left|h_{1}-h^{*}\right|^{2} k_{p}(t) \zeta(t) d t=0, \quad \forall \zeta \in C_{c}^{\infty}((0, T)) .
$$

Since, $k_{p} \neq 0, h^{*}=h_{1}$. 


\section{REFERENCES}

[1] M. Boulakia and S. Guerrero. A regularity result for a solid-fluid system associated to the compressible Navier-Stokes equations. Ann. Inst. H. Poincaré Anal. Non Linéaire, 26(3):777-813, 2009.

[2] Muriel Boulakia, Sergio Guerrero, and Takéo Takahashi. Well-posedness for the coupling between a viscous incompressible fluid and an elastic structure. Nonlinearity, 32(10):3548-3592, 2019.

[3] Haim Brezis. Functional analysis, Sobolev spaces and partial differential equations. Springer Science \& Business Media, 2010.

[4] Philippe G. Ciarlet. Mathematical elasticity. Vol. I, volume 20 of Studies in Mathematics and its Applications. North-Holland Publishing Co., Amsterdam, 1988. Three-dimensional elasticity.

[5] Nicolae Cîndea, Sorin Micu, Ionel Rovenţa, and Marius Tucsnak. Particle supported control of a fluid-particle system. J. Math. Pures Appl. (9), 104(2):311-353, 2015.

[6] B. Desjardins and M. J. Esteban. On weak solutions for fluid-rigid structure interaction: compressible and incompressible models. Comm. Partial Differential Equations, 25(7-8):1399-1413, 2000.

[7] S. Ervedoza, M. Hillairet, and C. Lacave. Long-time behavior for the two-dimensional motion of a disk in a viscous fluid. Comm. Math. Phys., 329(1):325-382, 2014.

[8] Lawrence C. Evans. Partial differential equations, volume 19 of Graduate Studies in Mathematics. American Mathematical Society, Providence, RI, 1998.

[9] Eduard Feireisl. On the motion of rigid bodies in a viscous compressible fluid. Arch. Ration. Mech. Anal., 167(4):281308, 2003.

[10] Eduard Feireisl and Šárka Nečasová. On the long-time behaviour of a rigid body immersed in a viscous fluid. Appl. Anal., 90(1):59-66, 2011.

[11] Bernhard H. Haak, Debayan Maity, Takéo Takahashi, and Marius Tucsnak. Mathematical analysis of the motion of a rigid body in a compressible Navier-Stokes-Fourier fluid. Mathematical News / Mathematische Nachrichten, 2018.

[12] Matthias Hieber and Miho Murata. The $L^{p}$-approach to the fluid-rigid body interaction problem for compressible fluids. Evol. Equ. Control Theory, 4(1):69-87, 2015.

[13] Akitaka Matsumura and Takaaki Nishida. Initial-boundary value problems for the equations of motion of compressible viscous and heat-conductive fluids. Comm. Math. Phys., 89(4):445-464, 1983.

[14] Alexandre Munnier and Enrique Zuazua. Large time behavior for a simplified $N$-dimensional model of fluid-solid interaction. Comm. Partial Differential Equations, 30(1-3):377-417, 2005.

[15] Yoshihiro Shibata and Miho Murata. On the global well-posedness for the compressible Navier-Stokes equations with slip boundary condition. J. Differential Equations, 260(7):5761-5795, 2016.

[16] Takéo Takahashi, Marius Tucsnak, and George Weiss. Stabilization of a fluid-rigid body system. J. Differential Equations, 259(11):6459-6493, 2015.

[17] Juan Luis Vázquez and Enrique Zuazua. Large time behavior for a simplified 1D model of fluid-solid interaction. Comm. Partial Differential Equations, 28(9-10):1705-1738, 2003. 